\title{
Cofactor selectivity in methylmalonyl-CoA mutase, a model cobamide-dependent enzyme
}

Olga M. Sokolovskaya ${ }^{1,2}$, Kenny C. Mok ${ }^{1}$, Jong Duk Park ${ }^{1,3}$, Jennifer L. A. Tran ${ }^{1,4}$, Kathryn A. Quanstrom $^{1,5}$, and Michiko E. Taga ${ }^{1^{*}}$

${ }^{1}$ Department of Plant \& Microbial Biology, University of California, Berkeley, Berkeley, CA 94720 USA

2 Department of Chemistry, University of California, Berkeley, Berkeley, CA 94720 USA

${ }^{3}$ Present address: Department of Chemistry, Princeton University, Princeton, NJ 08544 USA

4 Present address: Evidation Health, San Mateo, CA 94401 USA

${ }^{5}$ Present address: University of Michigan School of Medicine, Ann Arbor, MI 48109 USA

*Correspondence to taga@berkeley.edu 


\section{Abstract}

Cobamides, a uniquely diverse family of enzyme cofactors related to vitamin $\mathrm{B}_{12}$, are produced exclusively by bacteria and archaea but used in all domains of life. While it is widely accepted that cobamide-dependent organisms require specific cobamides for their metabolism, the biochemical mechanisms that make cobamides functionally distinct are largely unknown. Here, we examine the effects of cobamide structural variation on a model cobamide-dependent enzyme, methylmalonyl-CoA mutase (MCM). The in vitro binding affinity of MCM for cobamides can be dramatically influenced by small changes in the structure of the lower ligand of the cobamide, and binding selectivity differs between bacterial orthologs of MCM. In contrast, variations in the lower ligand have minor effects on MCM catalysis. Bacterial growth assays demonstrate that cobamide requirements of MCM in vitro largely correlate with in vivo cobamide dependence. This result underscores the importance of enzyme selectivity in the cobamidedependent physiology of bacteria.

\section{Introduction}

Cobalamin, commonly referred to as vitamin $B_{12}$, is a versatile enzyme cofactor used by organisms in all domains of life. In humans, cobalamin is essential for methionine synthesis and the breakdown of fatty acids, amino acids, and cholesterol [1, 2]. Bacteria and archaea additionally use cobalamin and related cofactors, cobamides, for deoxyribonucleotide synthesis [3], metabolism of various carbon and energy sources [4-17], synthesis of secondary metabolites [18-25], sensing light [26], and other processes [15-17, 27-32]. The finding that $86 \%$ of bacterial species encode at least one cobamide-dependent enzyme in their genome [33] demonstrates the prevalence of cobamide-dependent metabolisms. Widespread use of these cofactors can be attributed to their chemical versatility, as they facilitate challenging chemical reactions including radical-initiated rearrangements, methylation reactions, and reductive cleavage of chemical bonds [34, 35].

All cobamides share the same core structure (Figure 1): a corrin ring that coordinates a cobalt ion, a variable "upper" axial ligand ( $R$ in Figure 1$)$, and a pseudo-nucleotide that is covalently attached to the corrin ring through an aminopropanol linker [36] or an ethanolamine linker, in the case of nor-cobamides [37,38]. The major differences among cobamides are in the structure of the nucleotide base, more commonly referred to as the lower axial ligand for its ability to coordinate the central cobalt ion. In cobalamin, the lower ligand is 5,6dimethylbenzimidazole (Figure 1, boxed); in other cobamides, different benzimidazoles, phenolics, and purines constitute the lower ligand (Figure 2C) [39-43].

While cobamides containing different lower ligands share the same chemically reactive moieties, specifically the cobalt center and methyl or 5'-deoxyadenosyl upper axial ligands, they are nonetheless functionally distinct. Culture-based studies have shown that only a subset of cobamides supports a given bacterial metabolism, and uptake or production of other cobamides 
can inhibit growth [39, 44-49]. The requirements of bacteria for particular cobamides is notable given the diversity of cobamides present in host-associated and environmental samples [40-42], coupled with the absence of de novo cobamide biosynthesis in more than half of bacteria [33]. Despite the biological relevance of cobamide structure, and the prevalence of cobamide use among bacteria [33, 50-52], little is understood about the biochemical mechanisms by which cobamides differentially impact microbial physiology.

The effect of lower ligand structure on the biochemistry of cobamide-dependent enzymes has been studied to a limited extent. In "base-on" enzymes, the lower ligand base coordinates the central cobalt ion of the cobamide, as drawn in Figure 1 [53-55]. Because the lower ligand is part of the catalytic center of the enzyme, lower ligand structure can influence catalysis through a variety of mechanisms [56-58], and cobamides unable to form an intramolecular coordinate bond are catalytically inactive in base-on enzymes [59, 60]. In contrast, in "base-off" enzymes the lower ligand is bound by the enzyme more than $10 \AA$ away from the active site [20, 61-70]. In a subset of base-off enzymes, referred to as "base-off/Hison," a histidine residue from the protein coordinates the cobalt ion in place of the lower ligand $[61,63]$. Despite its distance from the reactive center, lower ligand structure affects the activity of base-off enzymes, as evidenced by the cobamide cofactor selectivity of methionine synthase [71], methylmalonyl-CoA mutase (MCM) [60, 72], reductive dehalogenases [49], and other enzymes [59, 72, 73]. However, the mechanisms by which lower ligand structure affects the biochemistry of base-off cobamide-dependent enzymes remain unclear.

As MCM is one of the most abundant cobamide-dependent enzymes in bacterial genomes [33], and one of the two cobamide-dependent enzymes in humans, we have chosen to study the cobamide selectivity of MCM as a model for base-off/His-on enzymes, all of which share a structurally conserved $\mathrm{B}_{12}$-binding domain $[63,74]$. MCM catalyzes the interconversion of $(R)$-methylmalonyl-CoA and succinyl-CoA, a bidirectional reaction used in propionate metabolism [12, 75, 76], catabolism of branched amino acids and odd-chain fatty acids [76, 77], polyhydroxybutyrate degradation [78], secondary metabolite biosynthesis [79], and autotrophic carbon dioxide fixation [4, 80]. MCM-dependent pathways have been harnessed industrially for the bioproduction of propionate, bioplastics, biofuels, and antibiotics [81-88].

The presence of a cobamide lower ligand is required for MCM activity, as evidenced by the observation that adenosylcobinamide, a cobamide intermediate lacking a lower ligand (Figure 1), does not support MCM activity in vitro [89]. Three studies provide evidence that MCM is selective for cobamides with particular lower ligands. First, MCM from Propionibacterium shermanii was found to have different apparent $\mathrm{K}_{\mathrm{M}}$ values for cobamides, increasing from AdoCbl to Ado[Bza]Cba to Ado[Ade]Cba (refer to Table 1 for full names of cobamides), and MCM from sheep had a higher apparent $\mathrm{K}_{\mathrm{M}}$ for Ado[Bza]Cba than AdoCbl [72]. Second, $P$. shermanii MCM had a lower apparent $\mathrm{K}_{\mathrm{M}}$ for Ado[Cre]Cba than AdoCbl [60]. Third, in Sinorhizobium meliloti bacteroids MCM activity was highest with AdoCbl, intermediate with Ado[Bza]Cba, and absent with Ado[Ade]Cba [90]. Each of these studies includes only one or two cobamides other than cobalamin, and understandably so; cobamides are difficult to obtain 
in high quantities and must be purified from large volumes of bacterial cultures. Because of this, the response of MCM orthologs to the full diversity of cobamides has not been explored, and the mechanistic basis of cobamide selectivity remains unclear.

To investigate the mechanisms by which diverse lower ligands affect MCM function, we conducted in vitro binding and activity assays with MCM from S. meliloti (SmMCM). We discovered major differences in the binding affinities of eight naturally occurring cobamides for SmMCM, while cobamide structure affected enzyme activity to a lesser extent. Using six additional cobamides, five of which are novel analogs that have not been observed in nature or described previously, we identified structural elements of lower ligands that are determinants of binding to SmMCM. To probe the hypothesis that enzyme selectivity influences bacterial growth, we characterized the cobamide dependence of $S$. meliloti growth in vivo. By bridging the results of in vitro biochemistry of three bacterial MCM orthologs and the cobamide-dependent growth phenotypes of $S$. meliloti, we have elucidated molecular factors that contribute to the cobamidedependent physiology of bacteria.

\section{Results}

Lower ligand structure influences cobamide binding to MCM.

We chose SmMCM as a model to examine how lower ligand structure influences MCM function based on previous work demonstrating its activity as a homodimer encoded by a single gene $[91,92]$. We purified eight naturally occurring cobamides for in vitro studies of this protein, and chemically adenosylated each cobamide to produce the biologically active form used by MCM for catalysis. Previous studies showed that binding of cobamides to $P$. shermanii MCM can be detected in vitro by measuring quenching of intrinsic protein fluorescence [89]. We found that the fluorescence of purified, His-tagged SmMCM also decreased in a dose-dependent manner when the protein was reconstituted with increasing concentrations of AdoCbl (Figure $2 A)$. The equilibrium dissociation constant $\left(K_{d}\right)$ derived from these measurements, $0.03 \pm 0.02$ $\mu \mathrm{M}$ (Figure $2 \mathrm{C}$ ), is 6-fold lower than the $K_{d}$ reported for $P$. shermanii MCM [89]. Ado-cobinamide also bound SmMCM, as was observed with $P$. shermanii MCM [89], albeit with over 10-fold reduced affinity compared to cobalamin (Figure $2 \mathrm{~A}, \mathrm{C}$ ).

We next measured binding of other benzimidazolyl cobamides to SmMCM and found that Ado[5-MeBza]Cba and Ado[Bza]Cba, the cobamides most structurally similar to AdoCbl, also bound the enzyme. However, the absence of one or two methyl groups, respectively, in the lower ligands of these cobamides caused a decrease in binding affinity relative to AdoCbl (Figure 2A, C). Strikingly, no binding of Ado[5-OHBza]Cba to SmMCM was detected at low micromolar concentrations. To rule out the possibility that Ado[5-OHBza]Cba binds SmMCM but does not cause a fluorescence quench, we used an alternative, filtration-based, binding assay and observed little to no binding of Ado[5-OHBza]Cba to SmMCM at micromolar concentrations (Figure S1A, B). 
We expanded our analysis of SmMCM-cobamide binding selectivity to include cobamides from other structural classes. Both of the phenolyl cobamides tested, Ado[Cre]Cba and Ado[Phe]Cba, bound SmMCM with affinities similar to those of cobalamin and other benzimidazolyl cobamides (Figure 2B, C). In contrast, the purinyl cobamides Ado[2-MeAde]Cba and Ado[Ade]Cba had lower affinities for SmMCM compared to most benzimidazolyl cobamides (Figure 2B, C): Ado[2-MeAde]Cba bound SmMCM with 20-fold lower affinity than cobalamin, and Ado[Ade]Cba did not bind to any significant extent at micromolar concentrations (verified by the filtration assay, Figure S1C). Interestingly, for all three classes of lower ligands, the presence of a methyl substituent promoted binding relative to other cobamides of the same structural class.

\section{Bacterial MCM orthologs have distinct selectivity.}

To test whether cofactor-binding selectivity is a general phenomenon across bacterial MCM orthologs, we compared the cobamide-binding profile of SmMCM to that of MCM orthologs from Escherichia coli (EcMCM) and Veillonella parvula (VpMCM). Activity of EcMCM with AdoCbl has been reported both in vivo and in vitro, although its physiological role in $E$. coli remains unclear $[82,93]$. Annotations for two MCM homologs are present in the genome of $V$. parvula, and we purified the one that exhibits MCM activity when expressed in S. meliloti (see Materials and Methods). Because S. meliloti produces cobalamin [94], E. coli produces [2MeAde]Cba when provided cobinamide [95], and V. parvula produces [Cre]Cba [96], we expected that each ortholog should have distinct cobamide selectivity. Indeed, EcMCM had highest affinity for its native cobamide, Ado[2-MeAde]Cba (Figure 3A, C). All other cobamides bound with 2- to 3-fold reduced affinities relative to Ado[2-MeAde]Cba. Similarly, VpMCM had a higher affinity for Ado[Cre]Cba, its native cobamide, than AdoCbl (Figure 3B, C). VpMCM also bound Ado[2-MeAde]Cba and Ado[Bza]Cba with similar affinity.

We constructed a sequence alignment of MCM orthologs from diverse organisms known to produce or use various cobamides, in search of amino acid residues that could account for differences in cobamide binding (Figure S2A). The $\mathrm{B}_{12}$-binding domains of diverse MCM orthologs had high overall amino acid identity (38-70\%). Cases of low identity correlated with differences in the structural configuration of $\mathrm{MCM}$, which occurs in different organisms as a homodimer [92, 93, 97, 98], heterodimer [61, 99-101], or heterotetramer [80, 102] (Figure S2B). We focused our analysis on residues immediately surrounding the lower ligand in the available crystal structure of Homo sapiens MCM [97] (HsMCM) (Figure S2A, triangles). For the most part, these residues are highly conserved between orthologs. Interestingly, however, HsMCM residues Phe638, Phe722, and Ala731, which are conserved in SmMCM, are substituted with the more polar residues Tyr, Tyr, and Ser, respectively, in EcMCM (Figure S2A), which has a higher affinity for purinyl cobamides. Introducing mutations in SmMCM and EcMCM to test the importance of these residues proved challenging, as it resulted in reduced protein solubility and overall impaired cobamide binding (data not shown). Whether or not these residues co-vary with 
cobamide selectivity across other MCM orthologs is difficult to interpret because the cobamide selectivity of MCM from most organisms is unknown.

The lower ligand of cobamides modulates MCM reaction kinetics.

We reconstituted SmMCM with saturating amounts of each of the four cobamides that bound with highest affinity and measured conversion of $(R)$-methylmalonyl-CoA to succinyl-CoA under steady state conditions. Interestingly, the substrate $\mathrm{K}_{\mathrm{M}}$ was nearly invariable among the cobamides tested (Figure 4). Turnover was highest with AdoCbl $\left(26 \pm 1 \mathrm{~s}^{-1}\right)$ and 2- to 3-fold lower with other cobamides. Thus, all of the cobamides tested supported SmMCM catalysis with modest differences in $k_{\text {cat }}$. This finding is consistent with a previous observation that adenosylcobinamide-GDP, a cobamide precursor with an extended nucleotide loop and a guanine base, supported activity of $P$. shermanii MCM with only slight catalytic impairment compared to AdoCbl [103].

MCM-dependent growth of S. meliloti correlates with the binding selectivity of SmMCM for benzimidazolyl and purinyl cobamides, but not phenolyl cobamides.

To assess whether the cobamide-dependent growth of $S$. meliloti reflects MCM selectivity as observed in vitro, we cultured S. meliloti under conditions that require MCM activity. Examination of metabolic pathways encoded in the S. meliloti genome using the KEGG database [104] suggests that the degradation of branched amino acids isoleucine and valine to succinyl-CoA, an intermediate of the citric acid cycle, requires MCM. Indeed, growth of $S$. meliloti on L-isoleucine and L-valine as the only carbon sources was dependent on the presence of the bhbA gene, which encodes MCM [91] (Figure S3).

We constructed an S. meliloti strain incapable of synthesizing cobalamin and lacking cobamide-dependent enzymes other than MCM to ensure that differential growth could be attributed solely to MCM selectivity for added cobamides (see Materials and Methods). We cultivated this strain with L-isoleucine and L-valine as sole carbon sources in medium supplemented with different cobamides in their cyanylated (CN) forms, which is the form typically used for in vivo growth assays. Under these growth conditions, the maximum growth yield $\left(\mathrm{OD}_{600}\right)$ achieved at high concentrations of all of the cobamides was indistinguishable (Figure S4A-G). However, the concentration of cobamides required to achieve half of the maximal $\mathrm{OD}_{600}\left(\mathrm{EC}_{50}\right)$ differed based on the cobamide provided (Figure 5). Consistent with the binding data, $\mathrm{CNCbl}$ had the lowest $\mathrm{EC}_{50}$ value. $\mathrm{EC}_{50}$ values for $\mathrm{CN}[\mathrm{Bza}] \mathrm{Cba}$ and $\mathrm{CN}[2-$ MeAde]Cba were 5-fold higher than $\mathrm{CNCbl}$, and other cobamides had $\mathrm{EC}_{50}$ values two orders of magnitude higher than $\mathrm{CNCbl}$.

With the notable exception of the phenolyl cobamides, differences in the $\mathrm{EC}_{50}$ values of cobamides in vivo qualitatively correlated with the binding selectivity that we observed in vitro (Figure 2). Among benzimidazolyl cobamides, $E_{50}$ values increased from cobalamin to [Bza]Cba to [5-OHBza]Cba, consistent with the relative binding affinities of these cobamides. Similarly, [2-MeAde]Cba, which had an intermediate binding affinity for SmMCM, had a lower 
$\mathrm{EC}_{50}$ value than [Ade]Cba, which did not bind to SmMCM at low micromolar concentrations in vitro. The ability of [5-OHBza]Cba and [Ade]Cba to support growth suggests that these cobamides can bind SmMCM at concentrations higher than those tested in vitro; a control experiment with an S. meliloti strain lacking MCM rules out the possibility that high concentrations of cobamides $(10 \mu \mathrm{M})$ abiotically enable growth on isoleucine and valine (Figure S3).

We considered the possibility that differences in cobamide internalization by S. meliloti could also influence the $\mathrm{EC}_{50}$ measurements shown in Figure 5. When $\mathrm{S}$. meliloti cultures were supplemented with equimolar amounts of $\mathrm{CNCbI}$, CN[Ade]Cba, or $\mathrm{CN}[\mathrm{Cre}] \mathrm{Cba}$, the concentration of cobalamin extracted from the cellular fraction was 2- to 3-fold higher than [Ade]Cba and 5- to 6-fold higher than [Cre]Cba (Figure S5A). This result suggests that cobamides are differentially internalized or retained by the cells. However, MCM-dependent growth does not correlate with intracellular cobamide concentrations, as intracellular concentrations of cobalamin comparable to those of [Ade]Cba and [Cre]Cba supported $S$. meliloti growth to high densities (Figure S5). Therefore, the high $\mathrm{EC}_{50}$ of $\mathrm{CN}$ [Ade]Cba relative to $\mathrm{CNCbl}$ is more likely attributable to enzyme selectivity. Additional factors that could explain the high $\mathrm{EC}_{50}$ values of the phenolyl cobamides are considered in the Discussion.

Identification of structural elements that interfere with cobamide binding.

Given the apparent importance of MCM cobamide binding selectivity for the cobamidedependent growth of $S$. meliloti, we pursued a more mechanistic understanding of how lower ligand structure affects cobamide binding. When cobamides are bound to MCM, the lower ligand is surrounded by protein residues $[61,97]$. Therefore, the reduced affinity of certain cobamides for the enzyme could be a result of exclusion of their lower ligands from this binding pocket because of steric or electrostatic repulsion. We hypothesized that the poor binding of the purinyl cobamides Ado[Ade]Cba and Ado[2-MeAde]Cba is due to the presence of the exocyclic amine (N10) based on several observations: 1) Ado[5-OHBza]Cba, which also contains a polar functional group, had impaired binding to SmMCM (Figure 2A, C). 2) In the crystal structure of of HsMCM [97], residues Phe722 and Ala731, which are conserved in SmMCM, would be expected to electrostatically occlude the exocyclic amine of [Ade]Cba (Figure S6A, asterisk). In contrast, polar residues Tyr and Ser occupy these positions in EcMCM, which has higher affinity for purinyl cobamides (Figure S2A). 3) Structural modeling of Ado[Ade]Cba bound to HsMCM, which shares $59 \%$ amino acid identity to SmMCM in the $\mathrm{B}_{12}$-binding domain, suggests a significant displacement of the adenine lower ligand relative to the position of the lower ligand of AdoCbl, accompanied by significant expansion of the lower ligand binding pocket, which would be an unlikely conformation for the protein to adopt (Figure S6B-D).

To test the importance of the exocyclic amine of adenine in cofactor exclusion, we produced an unsubstituted purinyl cobamide, Ado[Pur]Cba [39]. Ado[Pur]Cba also had low affinity for SmMCM (Figure 6A, B), suggesting that the exocyclic amine of adenine is not a major cause of binding exclusion. Consistent with this result, two novel benzimidazolyl 
cobamides, Ado[7-MeBza]Cba and Ado[7-AmBza]Cba, bound SmMCM with comparable affinities to other benzimidazolyl cobamides (Figure 6A, B), despite being functionalized at the position analogous to $\mathrm{N} 10$ of adenine. Rather, these results suggest that the presence of nitrogens in the six-membered ring of the lower ligand interferes with binding. To test this hypothesis directly, we produced three novel cobamide analogs that contain at least one nitrogen in the six-membered ring of the lower ligand base. Comparison of the binding of Ado[6MePur]Cba and Ado[7-MeBza]Cba (Figure 6A, B) supported a role of ring nitrogens in binding inhibition, and comparison of binding affinities between Ado[Bza]Cba and Ado[5-AzaBza]Cba (Figure 2A, C and Figure 6A, B, respectively), and between Ado[7-AmBza]Cba and Ado[3DeazaAde]Cba (Figure 6A, B), revealed that a single nitrogen atom in the six-membered ring of the lower ligand was sufficient to severely impair binding.

As it was recently discovered to be a naturally occurring cobamide [39], we tested the

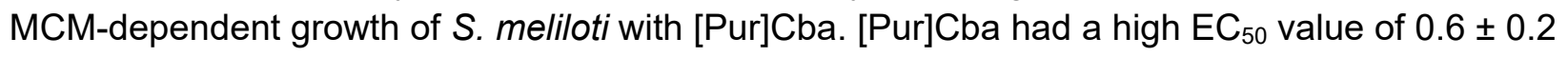
$\mu \mathrm{M}$ (Figure $\mathrm{S} 4 \mathrm{H}$ ), further supporting the correlation between binding and growth that we previously observed for benzimidazolyl and purinyl cobamides.

\section{Discussion}

Cobamides are distinct from other cofactors in their extensive structural diversity, with over a dozen forms that differ in the lower ligand base and nucleotide loop. How cobamide lower ligand structure influences the activity of cobamide-dependent enzymes has not been extensively explored. Here, we report a systematic analysis of the effects of cobamide lower ligand structure on the function of a model cobamide-dependent enzyme, MCM. Our results show that MCM exhibits varied affinities for different cobamides, and that this selectivity is linked to the physiology of the organism.

Our results show that the major determinant of cobamide selectivity in SmMCM is binding, with small changes in the lower ligand capable of dramatically altering the binding affinity of a cobamide. One explanation for these differences is that the chemical compatibility between the lower ligand base and the binding pocket of the protein strongly influences the binding affinity of cobamides; repulsion of the lower ligand on the basis of electrostatics could reduce the binding affinity of cobamides to MCM. While the structure of SmMCM has not been determined, a model generated by sequence alignment to HsMCM suggested a highly hydrophobic lower ligand binding pocket. Consistent with this, we observed higher affinity of cobamides with hydrophobic lower ligands to SmMCM, as well as interference of ring nitrogens with cobamide binding.

On the other hand, sequence alignments suggested that many of the hydrophobic residues predicted to immediately surround the lower ligand are conserved between diverse MCM orthologs that differ in cobamide selectivity. Assuming that the arrangement of the lower ligand binding pocket is similar across MCM orthologs, this suggests that interactions within the lower ligand binding pocket are not sufficient to account for selectivity. In a similar vein, 
examination of the residues surrounding the lower ligand in the cobamide-bound structures of reductive dehalogenases does not reveal the basis of exclusion of certain cobamides [49]. These observations suggest that the lower ligand may have an unknown role in the binding of cobamides to MCM. Consistent with this idea, studies of the kinetics and $\mathrm{pH}$ dependence of AdoCbl binding to $P$. shermanii MCM suggest a pre-association step, wherein a cofactor-protein complex is formed prior to displacement of the lower ligand of the cofactor by a histidine residue in the protein [89]. The nature of this complex is unknown, but potential interactions between the lower ligand and this conformation of the enzyme could provide an opportunity for lower ligand structure to impact the outcome of binding.

Our analysis of MCM orthologs from E. coli and V. parvula demonstrates that variations in cobamide selectivity have evolved in organisms with different physiologies. The cobamide selectivity patterns in the three MCM orthologs we examined correlate with the physiologies of the bacteria in two ways. First, in all three cases, each MCM ortholog has highest affinity for the native cobamide produced by the organism, suggesting that cobamide biosynthesis and selectivity of cobamide-dependent enzymes have coevolved. Second, SmMCM is more selective than EcMCM and VpMCM, and these differences in selectivity correlate with differences in cobamide biosynthesis, acquisition, and use in these organisms. S. meliloti synthesizes cobalamin de novo and is incapable of attaching purinyl and phenolyl lower ligands to cobamide precursors [96]. Thus, its cobamide-dependent enzymes have likely evolved to function best with cobalamin. In contrast, E. coli does not synthesize cobamides de novo and instead relies on the importer BtuBFCD to acquire cobamides from the environment $[105,106]$. Alternatively, $E$. coli can produce a variety of benzimidazolyl and purinyl cobamides when provided with precursors [95], making the ability to use multiple cobamides likely advantageous. Like S. meliloti, V. parvula synthesizes cobamides de novo, but can produce both benzimidazolyl and phenolyl cobamides $[96,107]$ and also encodes membrane transport components adjacent to cobalamin riboswitches [108], which are likely to be cobamide importers $[52,109]$. Thus, the ability of VpMCM to bind diverse cobamides is similarly consistent with its physiology.

Relative to cobamide binding selectivity, our results suggest that effects of lower ligand structure on the catalytic activity of MCM are minor. Among the cobamides we tested, the maximum differences in SmMCM turnover were 3-fold. We did not observe inhibition of MCM activity with any cobamides, in contrast to the strong inhibition that has been observed with analogs containing variations in the upper ligand or central metal, known as antivitamins [110112].

In addition to elucidating the biochemical basis of cobamide selectivity in MCM, a major aim of our work was to link biochemical selectivity with cobamide-dependent growth. Our results with benzimidazolyl and purinyl cobamides support the hypothesis that enzyme selectivity is a major determinant of cobamide-dependent growth. Interestingly, although phenolyl cobamides bound SmMCM with high affinity and supported catalysis in vitro, high concentrations were required to support growth of $S$. meliloti. This discrepancy can be partially explained by poorer 
internalization or retention of these cofactors as compared to cobalamin (Figure S5). The observation that the intracellular cobamide concentrations were 50- to 190-fold higher than the amount added to the growth medium (Figure S5A) suggests that cobamides could be internalized by an uptake mechanism that favors cobalamin, distinct from both BtuBFCD and ECF-CbrT $[113,114]$, both of which are absent from S. meliloti. Thus, we propose a model in which the cobamide-dependent growth of bacteria is influenced not only by binding selectivity of cobamide-dependent enzymes, but also by cobamide import (Figure 7). The lower effectiveness of phenolyl cobamides in supporting growth of $S$. meliloti could additionally be explained by inefficient adenosylation of these cobamides in vivo, as MCM requires the adenosyl upper axial ligand for activity. Whether or not adenosyltransferase enzymes, specifically CobA and PduO $[115,116]$ in $S$. meliloti, are selective with respect to lower ligand structure is unknown.

We and others have proposed the possibility of manipulating microbial communities using cobamides by taking advantage of the differential cobamide-dependent growth of bacteria $[39,117-119]$. Cobamides are predicted to mediate microbial interactions that are critical to the assembly of complex communities [33, 41, 45, 50, 120-123], so the ability to selectively inhibit or promote the growth of particular species using corrinoids with various lower ligands could be applied to alter the composition of microbial communities in ways that could promote environmental and human health. This possibility hinges on the ability to predict which cobamides support or inhibit growth of an organism of interest, which requires an understanding of the major biochemical determinants of growth. We observed here that the cobamide binding selectivity of a model base-off cobamide-dependent enzyme correlates with growth to a large extent. Thus, uncovering protein residues that confer selectivity would enable prediction of selectivity in cobamide-dependent enzymes, thereby facilitating prediction of the cobamide requirements of organisms of interest. Furthermore, our results suggest that additional steps of cobamide trafficking may be important determinants of cobamide-dependent growth. Future studies to understand how these various steps depend on cobamide structure will ultimately allow us to better understand, predict, and manipulate microbial interactions.

\section{Materials and Methods}

\section{Chemical reagents}

Chemicals were obtained from the following sources: 5'-chloro-5'-deoxyadenosine, Santa Cruz Biotechnology; 4-methylbenzimidazole, Accela; 5-methyl-1H-benzimidazole, ACROS Organics; phenol, J. T. Baker; zinc metal, Fisher Scientific; 5-methoxybenzimidazole, purine, and para-cresol, Alfa Aesar; methylmalonyl-CoA, methylmalonic acid, coenzyme A, adenosylcobalamin (coenzyme $\mathrm{B}_{12}$ ), cyanocobalamin, dicyanocobinamide, 6-methylpurine, $1 \mathrm{H}$ imidazo[4,5-c]pyridine-4-amine (3-deazaadenine), benzimidazole, adenine hemisulfate, 5azabenzimidazole, $1 \mathrm{H}$-benzo[d]imidazol-7-amine (4-aminobenzimidazole), 2-methyl-1H-purine6-amine (2-methyladenine), and bovine serum albumin (BSA), Sigma. 


\section{Molecular cloning, protein expression and purification}

SmMCM (locus SM_b20757, bhbA) was expressed from the pET28a vector, with an Nterminal hexahistidine (6xHis) tag, in E. coli BL21(DE3)pLysS (cloning primers listed in Table S1). The expression strain was grown to an optical density at $600 \mathrm{~nm}\left(\mathrm{OD}_{600}\right)$ of $0.6-0.8$ at 37 ${ }^{\circ} \mathrm{C}$, cooled on ice for $15 \mathrm{~min}$, and induced with $1 \mathrm{mM} \mathrm{IPTG}$ for $2.5 \mathrm{~h}$ at $37{ }^{\circ} \mathrm{C}$. Cells were lysed by sonication in $25 \mathrm{mM}$ Tris-HCl pH 8.0, $300 \mathrm{mM} \mathrm{NaCl}, 10 \mathrm{mM}$ imidazole, with $0.5 \mathrm{mM}$ PMSF, 1 $\mu \mathrm{g} / \mathrm{mL}$ leupeptin, $1 \mu \mathrm{g} / \mathrm{mL}$ pepstatin, and $1 \mathrm{mg} / \mathrm{mL}$ lysozyme. Clarified lysate was treated with $0.05 \%$ polyethyleneimine. An ÄKTA Pure 25 Fast Protein Liquid Chromatography (FPLC) system was used to purify the protein over a GE $5 \mathrm{~mL}$ HisTrap HF column, using a gradient of 21 to $230 \mathrm{mM}$ imidazole in the lysis buffer. Purified protein was dialyzed into $25 \mathrm{mM}$ Tris- $\mathrm{HCl} \mathrm{pH}$ 8.0, $300 \mathrm{mM} \mathrm{NaCl}, 10 \%$ glycerol and concentrated with a Vivaspin 10,000 MWCO protein concentrator. Final protein concentration was determined by $\mathrm{A}_{280}$ using the theoretical extinction coefficient $55810 \mathrm{M}^{-1} \mathrm{~cm}^{-1}$ [124]. EcMCM (locus b2917, scpA, previously sbmA) was expressed with an N-terminal 6xHis tag from a pET28a vector in E. coli BL21(DE3), by induction at $\mathrm{OD}_{600}$ 0.6-0.8 with $0.1 \mathrm{mM} \mathrm{IPTG}$, for $3.5 \mathrm{~h}$ at $30{ }^{\circ} \mathrm{C}$. The protein was purified as described above and the final concentration was determined by Coomassie-stained SDS-PAGE, using BSA as a standard.

The $V$. parvula genome has two MCM annotations: a heterotetramer (loci Vpar_RS06295, Vpar_RS06290) and a heterodimer (loci Vpar_RS09005, Vpar_RS09000). The functionality of both homologs was tested by complementation in S. meliloti. The two putative VpMCM enzymes were cloned into the pTH1227 vector and transferred by conjugation into an S. meliloti bhbA::Tn5 mutant. Complementation was assessed by growth in M9 liquid medium containing L-isoleucine and L-valine (see "S. meliloti growth assays" for additional details). S. meliloti co-expressing Vpar_RS09005 and Vpar_RS09000 showed identical growth to a strain expressing SmMCM from pTH1227 and was selected for in vitro studies.

The a subunit of VpMCM (encoded by Vpar_RS09005) was expressed with an Nterminal 6xHis tag from the pET-Duet expression vector in E. coli BL21(DE3). Protein expression was induced with $520 \mu \mathrm{M}$ IPTG for $6 \mathrm{~h}$ at $30{ }^{\circ} \mathrm{C}$. The protein was batch purified by nickel affinity and subsequently purified by FPLC using a HiTrapQ column with an $\mathrm{NaCl}$ gradient from 50 to $500 \mathrm{mM}$ in 20 Tris- $\mathrm{HCl}$ pH 8.0, 10\% glycerol. The $\beta$ subunit of VpMCM (encoded by Vpar_RS09000) was expressed separately with an N-terminal 6xHis tag from the pET-Duet expression vector in E. coli BL21(DE3). Expression was induced with $1 \mathrm{mM}$ IPTG for $22 \mathrm{~h}$ at 16 ${ }^{\circ} \mathrm{C}$ and the protein was purified using nickel-affinity chromatography as described for SmMCM. Purified protein was dialyzed into $25 \mathrm{mM}$ Tris- $\mathrm{HCl} \mathrm{pH} 8.0,300 \mathrm{mM} \mathrm{NaCl}, 10 \%$ glycerol, and 1 $\mathrm{mM} \beta$-mercaptoethanol. Concentration of $\alpha$ and $\beta$ subunits was determined by absorbance at $280 \mathrm{~nm}\left(\mathrm{~A}_{280}\right)$ using the theoretically calculated extinction coefficients $75290 \mathrm{M}^{-1} \mathrm{~cm}^{-1}$ and 74260 $\mathrm{M}^{-1} \mathrm{~cm}^{-1}$, respectively [124]. Equimolar amounts of $\alpha$ and $\beta$ subunits were combined during the setup of fluorescence binding assays.

E. coli thiokinase containing an $\mathrm{N}$-terminal $6 \mathrm{xH}$ is tag was expressed from a vector provided by Gregory Campanello from the laboratory of Ruma Banerjee. Expression was 
induced with $1 \mathrm{mM}$ IPTG in E. coli BL21(DE3)pLysS at $28{ }^{\circ} \mathrm{C}$ for $3 \mathrm{~h}$. The protein was purified as a heterodimer using nickel-affinity chromatography as described above. His-tagged Rhodopseudomonas palustris MatB [125] was expressed from a pET16b expression plasmid provided by Omer Ad from the laboratory of Michelle Chang. The protein was overexpressed in E. coli BL21(DE3) at $16{ }^{\circ} \mathrm{C}$ overnight, after induction with $1 \mathrm{mM} \mathrm{IPTG}$, and purified by nickel affinity chromatography as indicated above. Thiokinase and MatB concentrations were determined by Coomassie-stained SDS-PAGE, using BSA as a standard.

\section{Guided biosynthesis, extraction, and purification of cobamides}

Sporomusa ovata strain DSM 2662 was used for the production of its native cobamide, [Cre]Cba, and for production of [Phe]Cba, [5-MeBza]Cba, [Bza]Cba, [5-OHBza]Cba, [7MeBza]Cba, and [7-AmBza]Cba, by guided biosynthesis as previously described [44]. 5-OHBza was synthesized as described [96]. Salmonella enterica serovar Typhimurium strain LT2 and Propionibacterium acidipropionici strain DSM 20273 were used for production of [Ade]Cba [47, 126]. [2-MeAde]Cba, [Pur]Cba, [5-AzaBza]Cba, [3-DeazaAde]Cba, and [6-MePur]Cba were produced by guided biosynthesis in $P$. acidipropionici. Cobamides were extracted as previously described [47] and purified by High Performance Liquid Chromatography (HPLC) using previously published methods $[47,96,127]$ as well as additional methods listed in Table S2. In many cases more than one method was required to achieve high purity. Identity of cobamides was confirmed by Liquid Chromatography (LC) coupled to Mass Spectrometry (MS) using an Agilent 1260 LC/6120 quadrupole MS instrument. Lower ligand orientation in the novel cobamides [7-MeBza]Cba, [7-AmBza]Cba, [3-DeazaAde]Cba, and [6-MePur]Cba was inferred based on their absorbance spectra, which reveal a base-on conformation in the cyanylated form (Figure S7). The orientation of the lower ligands in [Pur]Cba and [5-AzaBza]Cba was not determined.

\section{Chemical adenosylation of cobamides}

Cobamide adenosylation was performed as previously described [127, 128]. Briefly, cobamides at concentrations $0.5-1 \mathrm{mM}$ were reduced with activated zinc metal under anaerobic conditions, with vigorous stirring for $0.5-2 \mathrm{~h}$. 5'-chloro-5'-deoxyadenosine was added and adenosylation was allowed to proceed for $1-3 \mathrm{~h}$ in the dark. The progress of the reaction was monitored by HPLC. Following adenosylation, cobamides were desalted using a C18 SepPak (Waters), purified by HPLC, desalted again, dried, and stored at $-20{ }^{\circ} \mathrm{C}$ or $-80^{\circ} \mathrm{C}$.

\section{Cobamide quantification}

Purified cobamides were dissolved in water and quantified by UV-Vis spectrophotometry on a BioTek Synergy 2 plate reader using the following extinction coefficients: for cyanylated benzimidazolyl cobamides, $\varepsilon_{518}=7.4 \times 10^{3} \mathrm{M}^{-1} \mathrm{~cm}^{-1}$ [129]; for cyanylated purinyl cobamides, $\varepsilon_{548}$ $=7.94 \times 10^{3} \mathrm{M}^{-1} \mathrm{~cm}^{-1}$ [130]; for cyanylated phenolyl cobamides, $\varepsilon_{495}=9.523 \times 10^{3} \mathrm{M}^{-1} \mathrm{~cm}^{-1}[131$ ]; for adenosylated benzimidazolyl cobamides (AdoCbl, Ado[5-MeBza]Cba, Ado[Bza]Cba, Ado[5- 
OHBza]Cba, Ado[7-MeBza]Cba, and Ado[7-AmBza]Cba), which are predominantly base-on in water, $\varepsilon_{522}=8.0 \mathrm{mM}^{-1} \mathrm{~cm}^{-1}$ [129]; for adenosylated purinyl and phenolyl cobamides (Ado[Ade]Cba, Ado[2-MeAde]Cba, Ado[Pur]Cba, Ado[Cre]Cba, and Ado[Phe]Cba), which are predominantly base-off in water, $\varepsilon_{458}=8.8 \mathrm{mM}^{-1} \mathrm{~cm}^{-1}$ [130]; for adenosylated azabenzimidazolyl cobamides (Ado[3-DeazaAde]Cba, Ado[5-AzaBza]Cba and Ado[6-MePur]Cba), which are a mixture of base-on and base-off in water, the concentration was estimated from the average of concentrations calculated using the extinction coefficients above.

\section{Fluorescence Binding Assays}

An in vitro assay previously described for measuring binding of AdoCbl to $P$. shermanii MCM [89] was adapted to a 96-well format: MCM $(0.2 \mu \mathrm{M})$ was combined with a range of cobamide concentrations (as specified in each experiment) in a black 96-well plate in $50 \mathrm{mM}$ potassium phosphate $\mathrm{pH} 7.5$ with $1 \mathrm{mM}$ DTT, on ice. All steps involving cobamides were conducted in the dark. The plate was centrifuged for $1 \mathrm{~min}$ at $3800 \mathrm{rpm}$ to level the surface of the liquid in each well. The plate was then incubated for $40 \mathrm{~min}$ at $30{ }^{\circ} \mathrm{C}$ to allow binding, with a brief shaking step after $30 \mathrm{~min}$. Preliminary experiments showed that this time is sufficient for equilibration. Following incubation, fluorescence emission at $340 \mathrm{~nm}$ ( $5 \mathrm{~nm}$ slit width) was measured upon excitation at $282 \mathrm{~nm}$ ( $5 \mathrm{~nm}$ slit width) using a Tecan Infinite M1000 PRO Plate Reader. Fluorescence, normalized to the initial value, was plotted as a function of cobamide concentration, and fit to the following equation [132]:

$$
\frac{F}{F_{0}}=1+\frac{\Delta F_{\max }}{F_{0}} \frac{\left([E]+[L]+K_{d}\right)-\sqrt{\left([E]+[L]+K_{d}\right)^{2}-4[E][L]}}{2[E]}
$$

where $F$ is fluorescence, $F_{0}$ is initial fluorescence, $[E]$ is total enzyme concentration, $[L]$ is total ligand concentration, and $K_{d}$ is the binding dissociation constant.

Filtration binding assay

Cobamides $(10 \mu \mathrm{M})$ with and without MCM $(15 \mu \mathrm{M})$ were incubated in $50 \mathrm{mM}$ Trisphosphate buffer $\mathrm{pH} 7.5$ at $30{ }^{\circ} \mathrm{C}$ for 40 min, transferred to Nanosep 10K Omega centrifugal devices (Pall Corporation), and centrifuged for 5 minutes at $13,900 \times \mathrm{g}$ to separate unbound cobamides from enzyme-bound cobamides. The UV-Vis spectra of the filtrates were recorded on a BioTek Synergy 2 plate reader.

\section{Structural modeling}

A model of SmMCM was generated using the Swiss-Model software [133] based on the known crystal structure of Homo sapiens MCM (HsMCM) (PBD ID: 2XIJ) [97]. No major differences were observed in the $\mathrm{B}_{12}$-binding domain between SmMCM models generated from HsMCM and Propionibacterium freudenrichii MCM (PDB ID: 4REQ) [61].

Maestro [134] was used to generate a model of HsMCM bound to [Ade]Cba. The initial structure of HsMCM bound to cobalamin (PDB ID: 2XIQ) [97] was prepared using standard methods. A constrained energy minimization (atoms within $10 \AA$ of cobalamin freely moving; atoms within a second $10 \AA$ shell constrained by a force constant of 200 ; remaining structure 
frozen) was performed using MacroModel [135]. The structure of the lower ligand was then modified to adenine, and the constrained energy minimization was repeated to generate a model of the lower ligand binding pocket bound to [Ade]Cba.

\section{Enzymatic synthesis of $(R)$-methylmalonyl-CoA}

(R)-methylmalonyl-CoA synthesis reactions contained the following in $10 \mathrm{~mL}: 100 \mathrm{mM}$ sodium phosphate $\mathrm{pH} 7.5,20 \mathrm{mM} \mathrm{MgCl}_{2}, 5 \mathrm{mM}$ ATP, $10 \mathrm{mM}$ methylmalonic acid, $2 \mathrm{mM}$ coenzyme A, $5 \mathrm{mM} \beta$-mercaptoethanol, and $1.5 \mu \mathrm{M}$ purified MatB protein. After combining ingredients on ice, the reaction was incubated at $37^{\circ} \mathrm{C}$ for $1 \mathrm{~h}$. The reaction was then frozen in liquid nitrogen and lyophilized. To purify $(R)$-methylmalonyl-CoA, the dried reaction mixture was resuspended in $3.2 \mathrm{~mL}$ water and the protein was precipitated with $200 \mu \mathrm{L}$ trichloroacetic acid; precipitate was pelleted; supernatant was neutralized with $200 \mu \mathrm{L}$ of $10 \mathrm{M} \mathrm{NaOH}$; and salts and remaining starting materials were removed using a C18 SepPak column (Waters) (loaded in $0.1 \%$ formic acid, washed with water, methylmalonyl-CoA eluted with $50 \%$ methanol in water). Formation of $(R)$-methylmalonyl-CoA was initially verified by ${ }^{1} \mathrm{H}$ NMR and in subsequent preparations by HPLC (Table S3). The concentration of (R)-methylmalonyl-CoA was determined using an extinction coefficient of $12.2 \mathrm{mM}^{-1} \mathrm{~cm}^{-1}$ at $259 \mathrm{~nm}$.

\section{MCM activity assays}

A thiokinase-coupled, spectrophotometric MCM activity assay was adapted from previous work [136], except that ADP was used instead of GDP, and the experiment was conducted in 96-well plates. Final concentrations of reagents in the assays are as follows: Trisphosphate buffer pH 7.5, 50 mM; DTNB, $400 \mu \mathrm{M}$; ADP, $1 \mathrm{mM}$; $\mathrm{MgCl}_{2}, 10 \mathrm{mM}$; (R)methylmalonyl-CoA, $0-4 \mathrm{mM}$; thiokinase, $5 \mu \mathrm{M}$; MCM, $50 \mathrm{nM}$; and cobamides, $2 \mu \mathrm{M}$. Preliminary experiments were conducted to ensure that concentrations of thiokinase, DTNB, and cobamides were not rate limiting.

Three separate mixes were prepared, all in 1X Tris-phosphate buffer: an assay mix containing DTNB, ADP, and $\mathrm{MgCl}_{2}$, a substrate mix containing (R)-methylmalonyl-CoA, and an enzyme mix containing thiokinase, MCM, and cobamides. All steps involving cobamides were conducted in the dark. The assay and enzyme mixes were prepared as a master mix and aliquoted into 96-well plates; substrate mixes were prepared in individual wells, in triplicate. All components were incubated at $30^{\circ} \mathrm{C}$ for 40 minutes to equilibrate temperature and allow prebinding of cobamides and MCM. After incubation, one replicate at a time, the substrate mix was added to the assay mix, followed by the enzyme mix. Absorbance at $412 \mathrm{~nm}\left(\mathrm{~A}_{412}\right)$ was recorded immediately after addition of enzyme and for 1-3 minutes, every 3 seconds, on a BioTek Synergy 2 plate reader. The increase in $A_{412}$ in reactions lacking substrate was subtracted from all readings, to account for reactivity of DTNB with thiols on protein surfaces. $A_{412}$ values were converted to concentration of free CoA using a pathlength correction determined for the reaction volume and extinction coefficient of $14150 \mathrm{M}^{-1} \mathrm{~cm}^{-1}$. 


\section{$\underline{\text { S. meliloti growth assays }}$}

MCM-dependent growth experiments were performed with S. meliloti strain Rm1021 cobD::gus $\mathrm{Gm}^{\mathrm{R}}$ metH::Tn5 $\Delta$ rdJ pMS03-nrdAB $\mathrm{Ec}^{+}$, which lacks cobamide-dependent enzymes other than MCM and does not synthesize cobalamin. $\operatorname{cobD}$ is required for cobalamin biosynthesis [137], metH encodes methionine synthase [137-139], and $n r d J$ encodes ribonucleotide reductase [140]. Because $n r d J$ is essential, the E. coli cobamide-independent ribonucleotide reductase encoded by $n r d A$ and $n r d B$ was expressed from the pMS03 plasmid [141]. The strain was pre-cultured in M9 medium [142] (modified concentration of $\mathrm{MgSO}_{4}: 1$ $\mathrm{mM}$ ) containing $0.1 \%$ sucrose, $2 \mathrm{~g} / \mathrm{L}$ isoleucine, $2 \mathrm{~g} / \mathrm{L}$ valine, $1 \mathrm{~g} / \mathrm{L}$ methionine, and $20 \mu \mathrm{g} / \mathrm{mL}$ gentamycin, shaking at $30{ }^{\circ} \mathrm{C}$. After two days, cells were washed and diluted to an $\mathrm{OD}_{600}$ of 0.02 into M9 medium containing $4 \mathrm{~g} / \mathrm{L}$ isoleucine, $4 \mathrm{~g} / \mathrm{L}$ valine, $1 \mathrm{~g} / \mathrm{L}$ methionine, $20 \mu \mathrm{g} / \mathrm{mL}$ gentamycin, and cobamides at various concentrations as indicated for each experiment, in 384well plates. The plates were incubated at $30{ }^{\circ} \mathrm{C}$ for $145 \mathrm{~h}$ in a Biotek Synergy 2 plate reader with linear shaking at $1140 \mathrm{cpm}$. $\mathrm{OD}_{600}$ was measured in $1 \mathrm{~h}$ increments.

For quantification of intracellular cobamides in $S$. meliloti, the strain above was precultured as described, diluted into $50 \mathrm{~mL}$ of M9 medium containing $0.2 \%$ sucrose and various cobamides, and grown for $48 \mathrm{~h}$ (until $\mathrm{OD}_{600}$ 0.6-0.8). Cobamides were extracted from cell pellets as previously described [47], using $5 \mathrm{~mL}$ of methanol containing $500 \mu \mathrm{g}$ of potassium cyanide, and including a partial purification by means of a wash step with $20 \%$ methanol in water during the SepPak desalting procedure. Extracted cobamides were quantified by HPLC using peak areas at $525 \mathrm{~nm}$ and external standard curves, and cellular cobamide concentrations were calculated assuming $8 \times 10^{8} \mathrm{cells} / \mathrm{mL}$ at $\mathrm{OD}_{600} 1.0$ and cellular volume of $1 \mathrm{\mu m}^{3}$.

\section{Acknowledgements}

We thank current and past members of the Taga lab and Ruma Banerjee, Judith Klinman, Susan Marqusee, and David Savage for helpful discussions; Amrita Hazra, Amanda Shelton, Alexa Nicolas, Zachary Hallberg, Kristopher Kennedy, Joseph Maa, and Judith Klinman for critical reading of the manuscript; Anna Beatrice Grimaldo for help with guided biosynthesis of cobamide analogs; Amrita Hazra and Florian Widner for providing 5-OHBza; Victoria Innocent for assisting with cobamide accumulation assays; Ruma Banerjee, Gregory Campanello, Michelle Chang, and Omer Ad for providing expression plasmids; Kathleen Durkin for help with molecular modeling; and Krishna Niyogi and Arash Komeili for use of their equipment. This work was supported by NIH grants R01GM114535 and DP2AI117984 to MET. OS was also supported by the NIH Chemical Biology Training Grant T32 GM066698, JT by the UCSF work study program, and modeling work at the UC Berkeley Molecular Graphics and Computation Facility by NIH S10OD023532. 


\section{Figures and Tables}

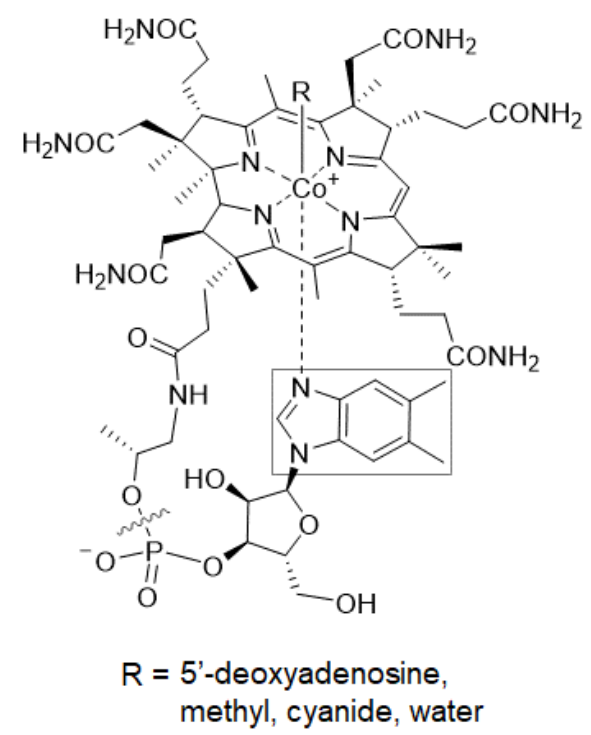

Figure 1: The structure of cobalamin. The lower ligand, boxed, varies in other cobamides. Cobinamide, a cobamide precursor, lacks a nucleotide base (delineated by the wavy line).

Table 1: Abbreviations used for cobamides and upper axial ligands.

\begin{tabular}{|c|c|}
\hline Abbreviation & Cobamide name \\
\hline $\mathrm{Cbl}$ & cobalamin \\
\hline [5-MeBza]Cba & 5-methylbenzimidazolylcobamide \\
\hline [Bza]Cba & benzimidazolylcobamide \\
\hline [5-OHBza]Cba & 5-hydroxybenzimidazolylcobamide \\
\hline [Cre]Cba & para-cresolylcobamide \\
\hline [Phe]Cba & phenolylcobamide \\
\hline [Ade]Cba & adeninylcobamide \\
\hline [2-MeAde]Cba & 2-methyladeninylcobamide \\
\hline [Pur]Cba & purinylcobamide \\
\hline [7-MeBza]Cba & 7-methylbenzimidazolylcobamide \\
\hline [7-AmBza]Cba & 7-aminobenzimidazolylcobamide \\
\hline [5-AzaBza]Cba & 5-azabenzimidazolylcobamide \\
\hline [3-DeazaAde]Cba & 3-deazaadeninylcobamide \\
\hline [6-MePur]Cba & 6-methylpurinylcobamide \\
\hline Prefix & Upper ligand \\
\hline Ado & 5'-deoxyadenosine \\
\hline $\mathrm{CN}$ & cyanide \\
\hline
\end{tabular}




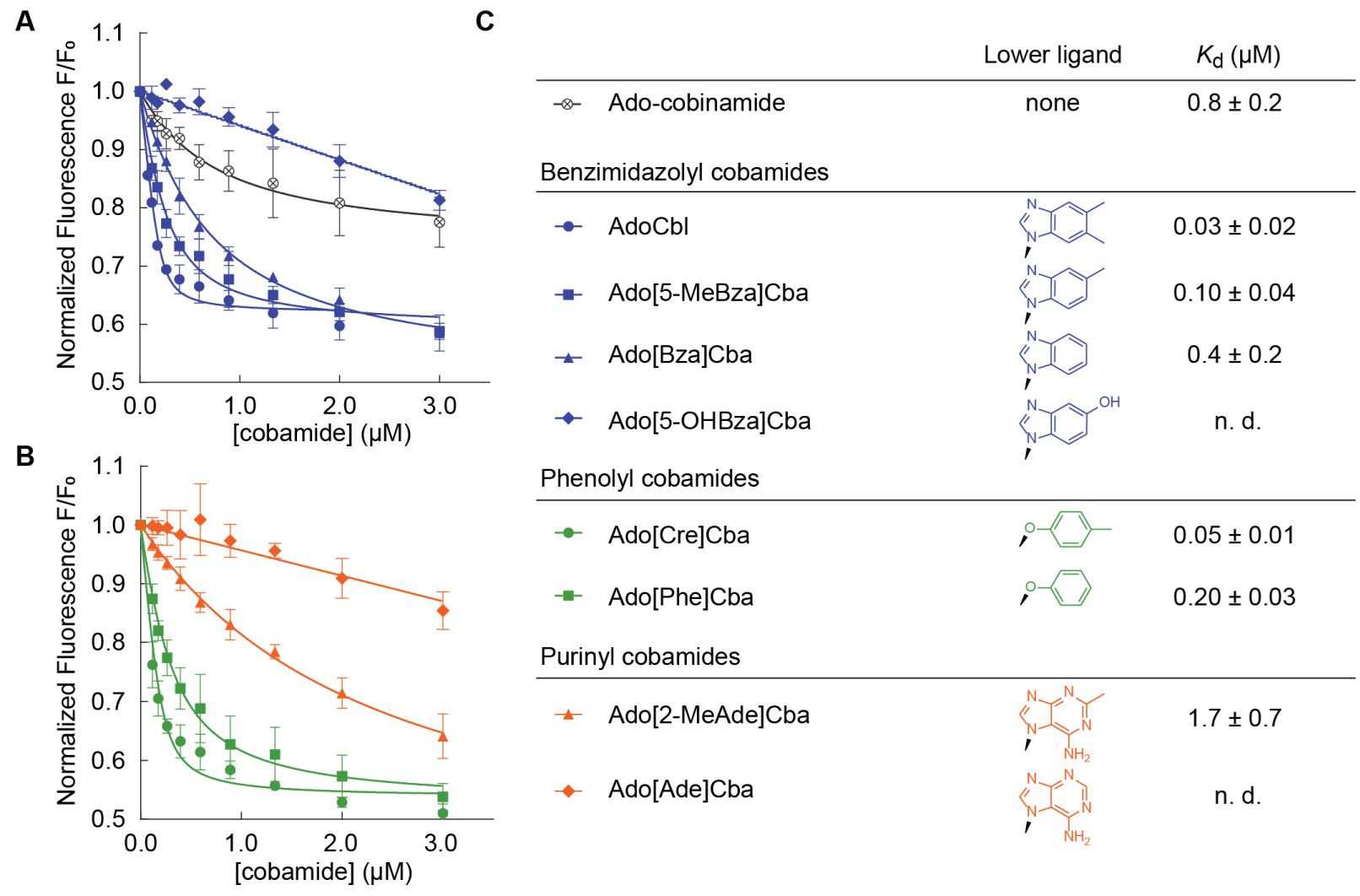

Figure 2: Binding of diverse cobamides to SmMCM (see also Figure S1). Fluorescence decrease of SmMCM when reconstituted with (A) benzimidazolyl cobamides (blue) and cobinamide (black), and (B) phenolyl (green) and purinyl (orange) cobamides. Data points represent the mean and standard deviation of three technical replicates from a single experiment. (C) $K_{d}$ values for different cobamides, reported as the average and standard deviation of three or more independent experiments, each consisting of technical triplicates. "n. d.," not determined, indicates that binding was too weak to determine $K_{\mathrm{d}}$.
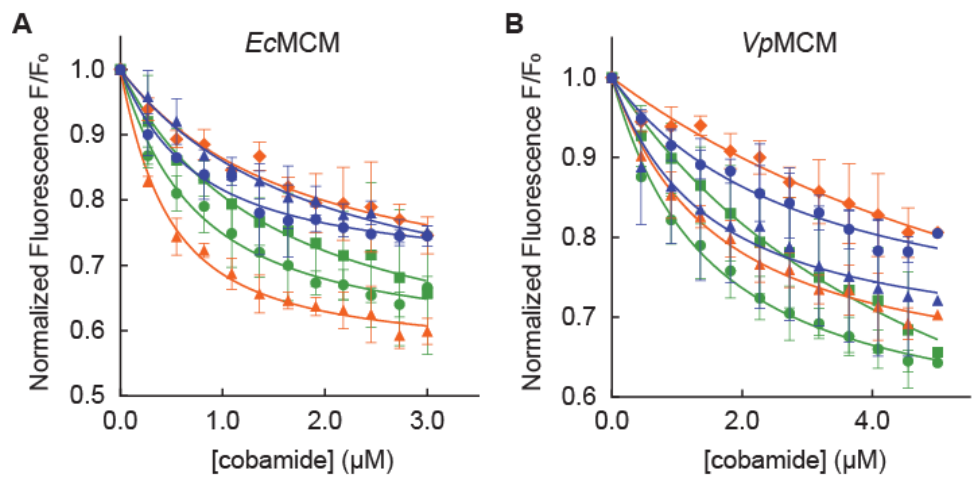

\begin{tabular}{lcc} 
C & \multicolumn{2}{c}{$K_{\mathrm{d}}(\mu \mathrm{M})$} \\
\cline { 2 - 3 } & $E c \mathrm{MCM}$ & $V p M C M$ \\
\hline$\rightarrow$ AdoCbl & $0.6 \pm 0.1$ & $2.8 \pm 0.6$ \\
$\rightarrow$ Ado[Bza]Cba & $1.8 \pm 0.2$ & $1.4 \pm 0.3$ \\
\hline$\rightarrow$ Ado[Cre]Cba & $0.62 \pm 0.09$ & $1.3 \pm 0.1$ \\
$\rightarrow$ Ado[Phe]Cba & $1.3 \pm 0.2$ & $5 \pm 1$ \\
\hline$\rightarrow$ Ado[2-MeAde]Cba & $0.35 \pm 0.04$ & $1.5 \pm 0.2$ \\
$\rightarrow$ Ado[Ade]Cba & $1.6 \pm 0.4$ & $9 \pm 4$
\end{tabular}

Figure 3: Binding selectivity of diverse MCM orthologs (see also Figure S2). Fluorescence binding assays with (A) E. coli MCM and (B) V. parvula MCM. Data points and error bars represent the mean and 
standard deviation, respectively, of technical triplicates from a single experiment; each replicate consisted of an independent cobamide dilution. $K_{d}$ values from the fitted curves in $(A)$ and $(B)$ are reported in $(C)$; error values reflect the standard error of the curve fit. $K_{d}$ values for VpMCM binding to Ado[Cre]Cba and AdoCbl and for EcMCM binding to all cobamides were reproduced in independent experiments.

A

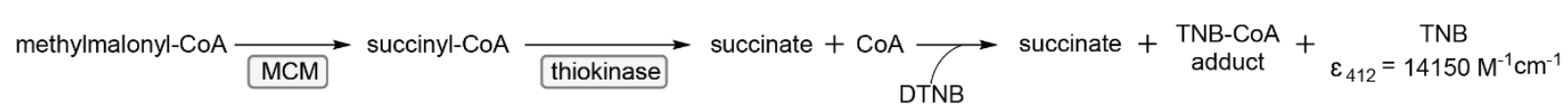

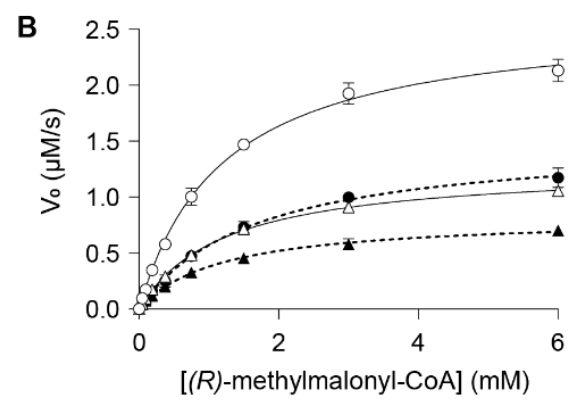

C

\begin{tabular}{lccc} 
Benzimidazolyl cobamides & $k_{\text {cat }}\left(\mathrm{s}^{-1}\right)$ & $K_{\mathrm{M}}(\mathrm{mM})$ & $k_{\text {cat }} / K_{\mathrm{M}}\left(\mathrm{mM}^{-1} \mathrm{~s}^{-1}\right)$ \\
\hline$\multimap-$ AdoCbl & $26 \pm 1$ & $1.2 \pm 0.1$ & $22 \pm 2$ \\
$-\triangle$ Ado[5-MeBza]Cba & $12.8 \pm 0.5$ & $1.2 \pm 0.1$ & $10 \pm 1$ \\
Phenolyl cobamides & & & \\
\hline$\cdots \bullet-$ Ado[Cre]Cba & $15 \pm 1$ & $1.66 \pm 0.05$ & $9 \pm 1$ \\
$\cdots$-.- Ado[Phe]Cba & $8.3 \pm 0.4$ & $1.2 \pm 0.1$ & $7 \pm 1$
\end{tabular}

Figure 4: Activity of Sinorhizobium meliloti MCM with different cobamide cofactors. (A) Succinyl-CoA formation was detected using a coupled spectrophotometric assay [136]. DTNB, dithionitrobenzoate (Ellman's Reagent); TNB, thionitrobenzoate; CoA, Coenzyme A. (B) Michaelis-Menten kinetic analysis of SmMCM reconstituted with various cobamides. Data points and error bars represent the mean and standard deviation, respectively, of three technical replicates from one experiment; each replicate consisted of an independent substrate dilution. Kinetic constants are presented in (C).

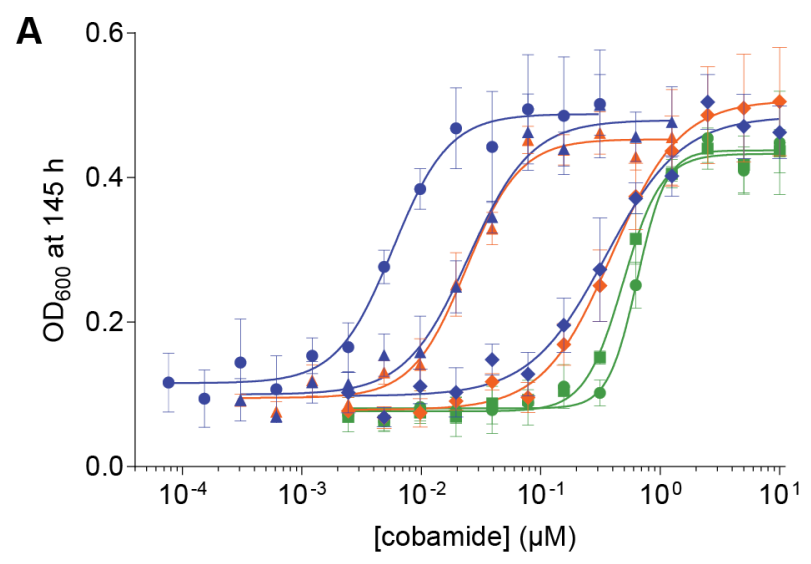
B

\begin{tabular}{lc} 
Benzimidazolyl cobamides & $\mathrm{EC}_{50}(\mu \mathrm{M})$ \\
\hline$\bullet \quad \mathrm{CNCbl}$ & $0.005 \pm 0.001$ \\
$\star \mathrm{CN}[\mathrm{Bza}] \mathrm{Cba}$ & $0.027 \pm 0.007$ \\
$\leftarrow \mathrm{CN}[5-\mathrm{OHBza}] \mathrm{Cba}$ & $0.4 \pm 0.1$
\end{tabular}
Phenolyl cobamides

$\begin{array}{ll}\rightarrow \mathrm{CN}[\mathrm{Cre}] \mathrm{Cba} & 0.7 \pm 0.1 \\ \rightarrow \mathrm{CN}[\mathrm{Phe}] \mathrm{Cba} & 0.4 \pm 0.1\end{array}$
Purinyl cobamides

$\begin{array}{lc}\rightarrow \text { CN[2-MeAde]Cba } & 0.024 \pm 0.009 \\ \rightarrow \text { CN[Ade]Cba } & 0.35 \pm 0.09\end{array}$

Figure 5: MCM-dependent growth of S. meliloti cobD::gus $\mathrm{Gm}^{\mathrm{R}}$ metH::Tn5 $\Delta$ rdJ pMS03-nrdABEEc ${ }^{+}$with various cobamides (see also Figures S3, S4, S5). (A) Dose dependence of growth based on OD600 at 145 
h. Data points and error bars represent the mean and standard deviation, respectively, of three biological replicates from a single experiment. $\mathrm{EC}_{50}$ values reported in $(\mathrm{B})$ are the average and standard deviation of five or more biological replicates across two or more independent experiments.

A

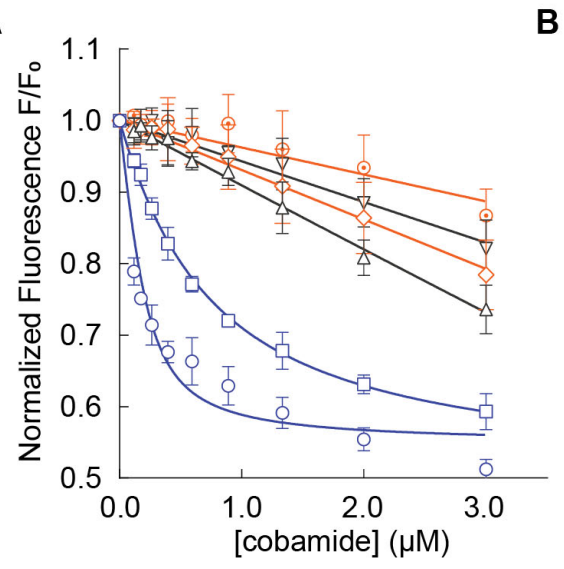

B

Cobamide analog
$-\odot \quad$ Ado[Pur]Cba*
$-\circ \quad$ Ado[7-MeBza]Cba
$-\square \quad$ Ado[7-AmBza]Cba
$\checkmark \quad$ Ado[6-MePur]Cba
$-\neg \quad$ Ado[5-AzaBza]Cba
$-\quad$ Ado[3-DeazaAde]Cba

Lower ligand

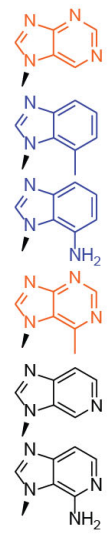

$K_{\mathrm{d}}(\mu \mathrm{M})$

n. d.

$0.10 \pm 0.02$

$0.7 \pm 0.1$

n. d.

n. d.

n. d.

Figure 6: Binding of cobamide analogs to SmMCM (see also Figure S7). (A) Fluorescence decrease of SmMCM when reconstituted with benzimidazolyl (blue), purinyl (orange), and azabenzimidazolyl (black) cobamide analogs. Data points represent the mean and standard deviation of three technical replicates from a single experiment. (B) $K_{d}$ values for different cobamides, reported as the average and standard deviation of three or more independent experiments, each consisting of technical triplicates. "n. d.," not determined, indicates that binding was too weak to determine $K_{\mathrm{d}}$. ${ }^{*}$ While it was unreported at the time of our study, [Pur]Cba was discovered to be the cobamide naturally produced by Desulfitobacterium hafniense [39].
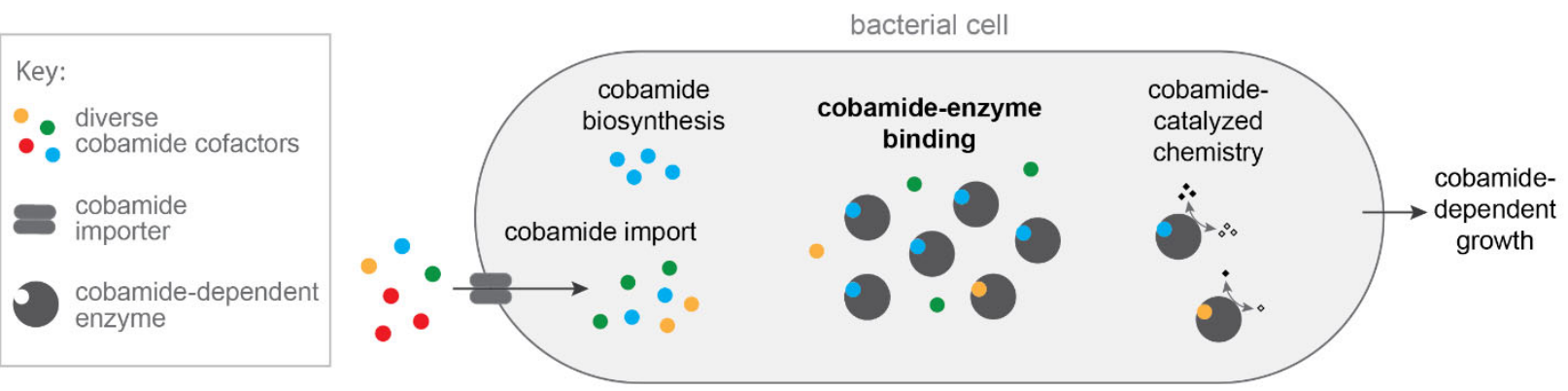

Figure 7: Model describing biochemical determinants of cobamide-dependent growth in bacteria. Cobamides differentially impact bacterial growth due to selective cobamide import and biosynthesis, cobamide-binding selectivity of cobamide-dependent enzymes, and cobamide-dependent catalysis. For MCM-dependent growth of $S$. meliloti, cobamide-binding selectivity is most strongly correlated with cobamide-dependent growth of the organism. 


\section{References}

1. Brodie JD, Burke GT, Mangum JH. 1970. Methylcobalamin as an intermediate in mammalian methionine biosynthesis. Biochemistry 9:4297-4302.

2. Fenton WA, Hack AM, Willard HF, Gertler A, Rosenberg LE. 1982. Purification and properties of methylmalonyl coenzyme A mutase from human liver. Arch Biochem Biophys 214:815-23.

3. Licht S, Gerfen GJ, Stubbe J. 1996. Thiyl radicals in ribonucleotide reductases. Science 271:477-81.

4. $\quad$ Berg IA, Kockelkorn D, Buckel W, Fuchs G. 2007. A 3-hydroxypropionate/4hydroxybutyrate autotrophic carbon dioxide assimilation pathway in Archaea. Sci 318:1782-6.

5. Ljungdahl LG. 1986. The autotrophic pathway of acetate synthesis in acetogenic bacteria. Annu Rev Microbiol 40:415-50.

6. Krasotkina J, Walters T, Maruya KA, Ragsdale SW. 2001. Characterization of the $B_{12-}$ and iron-sulfur-containing reductive dehalogenase from Desulfitobacterium chlororespirans. J Biol Chem 276:40991-7.

7. Scarlett FA, Turner JM. 1976. Microbial metabolism of amino alcohols. Ethanolamine catabolism mediated by coenzyme $\mathrm{B}_{12}$-dependent ethanolamine ammonia-lyase in Escherichia coli and Klebsiella aerogenes. J Gen Microbiol 95:173-6.

8. Stupperich E, Konle R. 1993. Corrinoid-dependent methyl transfer reactions are involved in methanol and 3,4-dimethoxybenzoate metabolism by Sporomusa ovata. Appl Environ Microbiol 59:3110-6.

9. Jeter RM. 1990. Cobalamin-dependent 1,2-propanediol utilization by Salmonella typhimurium. J Gen Microbiol 136:887-96.

10. Forage RG, Foster MA. 1982. Glycerol fermentation in Klebsiella pneumoniae: functions of the coenzyme $\mathrm{B}_{12}$-dependent glycerol and diol dehydratases. J Bacteriol 149:413-9.

11. Erb TJ, Retey J, Fuchs G, Alber BE. 2008. Ethylmalonyl-CoA mutase from Rhodobacter sphaeroides defines a new subclade of coenzyme $\mathrm{B}_{12}$-dependent acyl-CoA mutases. $\mathrm{J}$ Biol Chem 283:32283-93.

12. Vrijbloed JW, Zerbe-Burkhardt K, Ratnatilleke A, Grubelnik-Leiser A, Robinson JA. 1999. Insertional inactivation of methylmalonyl coenzyme A (CoA) mutase and isobutyryl-CoA mutase genes in Streptomyces cinnamonensis: influence on polyketide antibiotic biosynthesis. J Bacteriol 181:5600-5.

13. Korotkova N, Chistoserdova L, Kuksa V, Lidstrom ME. 2002. Glyoxylate regeneration pathway in the methylotroph Methylobacterium extorquens AM1. Journal of Bacteriology 184:1750-1758.

14. Ferguson DJ, Jr., Krzycki JA. 1997. Reconstitution of trimethylamine-dependent coenzyme M methylation with the trimethylamine corrinoid protein and the isozymes of methyltransferase II from Methanosarcina barkeri. J Bacteriol 179:846-52. 
15. Barker HA. 1985. beta-Methylaspartate-glutamate mutase from Clostridium tetanomorphum. Methods Enzymol 113:121-33.

16. Chang $\mathrm{CH}$, Frey PA. 2000. Cloning, sequencing, heterologous expression, purification, and characterization of adenosylcobalamin-dependent D-lysine 5,6-aminomutase from Clostridium sticklandii. J Biol Chem 275:106-14.

17. Chen HP, Wu SH, Lin YL, Chen CM, Tsay SS. 2001. Cloning, sequencing, heterologous expression, purification, and characterization of adenosylcobalamin-dependent Dornithine aminomutase from Clostridium sticklandii. J Biol Chem 276:44744-50.

18. Allen KD, Wang SC. 2014. Initial characterization of Fom3 from Streptomyces wedmorensis: The methyltransferase in fosfomycin biosynthesis. Arch Biochem Biophys 543:67-73.

19. Blaszczyk AJ, Silakov A, Zhang B, Maiocco SJ, Lanz ND, Kelly WL, Elliott SJ, Krebs C, Booker SJ. 2016. Spectroscopic and electrochemical characterization of the iron-sulfur and cobalamin cofactors of TsrM, an unusual radical S-adenosylmethionine methylase. J Am Chem Soc 138:3416-26.

20. Bridwell-Rabb J, Zhong A, Sun HG, Drennan CL, Liu HW. 2017. A B 12 -dependent radical SAM enzyme involved in oxetanocin-A biosynthesis. Nature 544:322-326.

21. Kim HJ, Liu YN, McCarty RM, Liu HW. 2017. Reaction catalyzed by GenK, a cobalamindependent radical S-adenosyl-L-methionine methyltransferase in the biosynthetic pathway of gentamicin, proceeds with retention of configuration. J Am Chem Soc 139:16084-16087.

22. Pierre S, Guillot A, Benjdia A, Sandstrom C, Langella P, Berteau O. 2012. Thiostrepton tryptophan methyltransferase expands the chemistry of radical SAM enzymes. Nat Chem Biol 8:957-9.

23. Werner WJ, Allen KD, Hu K, Helms GL, Chen BS, Wang SC. 2011. In vitro phosphinate methylation by PhpK from Kitasatospora phosalacinea. Biochemistry 50:8986-8.

24. Marous DR, Lloyd EP, Buller AR, Moshos KA, Grove TL, Blaszczyk AJ, Booker SJ, Townsend CA. 2015. Consecutive radical S-adenosylmethionine methylations form the ethyl side chain in thienamycin biosynthesis. Proc Natl Acad Sci U S A 112:10354-8.

25. Jung WS, Kim E, Yoo YJ, Ban YH, Kim EJ, Yoon YJ. 2014. Characterization and engineering of the ethylmalonyl-CoA pathway towards the improved heterologous production of polyketides in Streptomyces venezuelae. Appl Microbiol Biotechnol 98:3701-13.

26. Ortiz-Guerrero JM, Polanco MC, Murillo FJ, Padmanabhan S, Elias-Arnanz M. 2011. Light-dependent gene regulation by a coenzyme $B_{12}$-based photoreceptor. Proc Natl Acad Sci U S A 108:7565-70.

27. Gough SP, Petersen BO, Duus JO. 2000. Anaerobic chlorophyll isocyclic ring formation in Rhodobacter capsulatus requires a cobalamin cofactor. Proc Natl Acad Sci U S A 97:6908-13. 
28. Parks JM, Johs A, Podar M, Bridou R, Hurt RA, Jr., Smith SD, Tomanicek SJ, Qian Y, Brown SD, Brandt CC, Palumbo AV, Smith JC, Wall JD, Elias DA, Liang L. 2013. The genetic basis for bacterial mercury methylation. Science 339:1332-5.

29. Miles ZD, McCarty RM, Molnar G, Bandarian V. 2011. Discovery of epoxyqueuosine (oQ) reductase reveals parallels between halorespiration and tRNA modification. Proc Natl Acad Sci U S A 108:7368-72.

30. Yaneva N, Schuster J, Schäfer F, Lede V, Przybylski D, Paproth T, Harms H, Müller RH, Rohwerder T. 2012. Bacterial acyl-CoA mutase specifically catalyzes coenzyme $B_{12}-$ dependent isomerization of 2-hydroxyisobutyryl-CoA and (S)-3-hydroxybutyryl-CoA. J Biol Chem 287:15502-11.

31. Cracan V, Banerjee R. 2012. Novel coenzyme $B_{12}$-dependent interconversion of isovaleryl-CoA and pivalyl-CoA. J Biol Chem 287:3723-32.

32. Romine MF, Rodionov DA, Maezato Y, Anderson LN, Nandhikonda P, Rodionova IA, Carre A, Li X, Xu C, Clauss TR, Kim YM, Metz TO, Wright AT. 2017. Elucidation of roles for vitamin $B_{12}$ in regulation of folate, ubiquinone, and methionine metabolism. Proc Natl Acad Sci U S A 114:E1205-e1214.

33. Shelton AN, Seth EC, Mok KC, Han AW, Jackson SN, Haft DR, Taga ME. 2019. Uneven distribution of cobamide biosynthesis and dependence in bacteria predicted by comparative genomics. Isme j 13:789-804.

34. Banerjee R, Ragsdale SW. 2003. The many faces of vitamin $\mathrm{B}_{12}$ : Catalysis by cobalamin-dependent enzymes. Annu Rev Biochem 72:209-247.

35. Bridwell-Rabb J, Drennan CL. 2017. Vitamin $B_{12}$ in the spotlight again. Curr Opin Chem Biol 37:63-70.

36. Hodgkin DC, Kamper J, Mackay M, Pickworth J, Trueblood KN, White JG. 1956. Structure of vitamin $\mathrm{B}_{12}$. Nature 178:64-66.

37. Kräutler B, Fieber W, Ostermann S, Fasching M, Ongania K-H, Gruber K, Kratky C, Mikl C, Siebert A, Diekert G. 2003. The cofactor of tetrachloroethene reductive dehalogenase of Dehalospirillum multivorans is norpseudo- $\mathrm{B}_{12}$, a new type of a natural corrinoid, vol 86.

38. Keller S, Treder A, von Reuss SH, Escalante-Semerena JC, Schubert T. 2016. The SMUL_1544 gene product governs norcobamide biosynthesis in the tetrachloroethenerespiring bacterium Sulfurospirillum multivorans. J Bacteriol 198:2236-43.

39. Yan J, Bi M, Bourdon AK, Farmer AT, Wang PH, Molenda O, Quaile AT, Jiang N, Yang Y, Yin Y, Simsir B, Campagna SR, Edwards EA, Loffler FE. 2018. Purinyl-cobamide is a native prosthetic group of reductive dehalogenases. Nat Chem Biol 14:8-14.

40. Allen RH, Stabler SP. 2008. Identification and quantitation of cobalamin and cobalamin analogues in human feces. Am J Clin Nutr 87:1324-35.

41. Men Y, Seth EC, Yi S, Crofts TS, Allen RH, Taga ME, Alvarez-Cohen L. 2015. Identification of specific corrinoids reveals corrinoid modification in dechlorinating microbial communities. Environ Microbiol 17:4873-84. 
42. Girard CL, Santschi DE, Stabler SP, Allen RH. 2009. Apparent ruminal synthesis and intestinal disappearance of vitamin $\mathrm{B}_{12}$ and its analogs in dairy cows. J Dairy Sci 92:4524-9.

43. Renz P. 1999. Biosynthesis of the 5,6-dimethylbenzimidazole moiety of cobalamin and of the other bases found in natural corrinoids, p 557-566. In Banerjee R (ed), Chemistry and Biochemistry of B12. John Wiley \& Sons, Inc., New York.

44. Mok KC, Taga ME. 2013. Growth inhibition of Sporomusa ovata by incorporation of benzimidazole bases into cobamides. J Bacteriol 195:1902-11.

45. Helliwell KE, Lawrence AD, Holzer A, Kudahl UJ, Sasso S, Krautler B, Scanlan DJ, Warren MJ, Smith AG. 2016. Cyanobacteria and eukaryotic algae use different chemical variants of vitamin $\mathrm{B}_{12}$. Curr Biol 26:999-1008.

46. Watanabe F, Nakano Y, Stupperich E. 1992. Different corrinoid specificities for cell growth and cobalamin uptake in Euglena gracilis Z, vol 138.

47. Yi S, Seth EC, Men YJ, Stabler SP, Allen RH, Alvarez-Cohen L, Taga ME. 2012. Versatility in corrinoid salvaging and remodeling pathways supports corrinoid-dependent metabolism in Dehalococcoides mccartyi. Appl Environ Microbiol 78:7745-52.

48. Yan J, Simsir B, Farmer AT, Bi M, Yang Y, Campagna SR, Loffler FE. 2016. The corrinoid cofactor of reductive dehalogenases affects dechlorination rates and extents in organohalide-respiring Dehalococcoides mccartyi. Isme j 10:1092-101.

49. Keller S, Kunze C, Bommer M, Paetz C, Menezes RC, Svatoš A, Dobbek H, Schubert T. 2018. Selective utilization of benzimidazolyl-norcobamides as cofactors by the tetrachloroethene reductive dehalogenase of Sulfurospirillum multivorans. Journal of Bacteriology doi:10.1128/jb.00584-17.

50. Degnan PH, Barry NA, Mok KC, Taga ME, Goodman AL. 2014. Human gut microbes use multiple transporters to distinguish vitamin $\mathrm{B}_{12}$ analogs and compete in the gut. Cell Host Microbe 15:47-57.

51. Zhang Y, Rodionov DA, Gelfand MS, Gladyshev VN. 2009. Comparative genomic analyses of nickel, cobalt and vitamin $B_{12}$ utilization. BMC Genomics 10:78.

52. Rodionov DA, Vitreschak AG, Mironov AA, Gelfand MS. 2003. Comparative genomics of the vitamin $B_{12}$ metabolism and regulation in prokaryotes. J Biol Chem 278:41148-59.

53. Yamanishi M, Yunoki M, Tobimatsu T, Sato H, Matsui J, Dokiya A, luchi Y, Oe K, Suto K, Shibata N, Morimoto Y, Yasuoka N, Toraya T. 2002. The crystal structure of coenzyme $\mathrm{B}_{12}$-dependent glycerol dehydratase in complex with cobalamin and propane1,2-diol. Eur J Biochem 269:4484-94.

54. Larsson KM, Logan DT, Nordlund P. 2010. Structural basis for adenosylcobalamin activation in AdoCbl-dependent ribonucleotide reductases. ACS Chem Biol 5:933-42.

55. Shibata N, Sueyoshi Y, Higuchi Y, Toraya T. 2018. Direct participation of a peripheral side chain of a corrin ring in coenzyme $B_{12}$ catalysis. Angew Chem Int Ed Engl 57:78307835 . 
56. Conrad KS, Jordan CD, Brown KL, Brunold TC. 2015. Spectroscopic and computational studies of cobalamin species with variable lower axial ligation: implications for the mechanism of Co-C bond activation by class I cobalamin-dependent isomerases. Inorg Chem 54:3736-47.

57. Brown KL. 2006. The enzymatic activation of coenzyme $B_{12}$. Dalton Transactions doi:10.1039/B517599M:1123-1133.

58. Kozlowski PM, Zgierski MZ. 2004. Electronic and steric influence of trans axial base on the stereoelectronic properties of cobalamins. The Journal of Physical Chemistry $B$ 108:14163-14170.

59. Poppe L, Bothe H, Bröker G, Buckel W, Stupperich E, Rétey J. 2000. Elucidation of the coenzyme binding mode of further $\mathrm{B}_{12}$-dependent enzymes using a base-off analogue of coenzyme $\mathrm{B}_{12}$, vol 10.

60. Poppe L, Stupperich E, Hull WE, Buckel T, Retey J. 1997. A base-off analogue of coenzyme- $\mathrm{B}_{12}$ with a modified nucleotide loop; ${ }^{1} \mathrm{H}-\mathrm{NMR}$ structure analysis and kinetic studies with $(R)$-methylmalonyl-CoA mutase, glycerol dehydratase, and diol dehydratase. Eur J Biochem 250:303-7.

61. Mancia F, Keep NH, Nakagawa A, Leadlay PF, McSweeney S, Rasmussen B, Bosecke $P$, Diat O, Evans PR. 1996. How coenzyme $B_{12}$ radicals are generated: the crystal structure of methylmalonyl-coenzyme A mutase at 2 A resolution. Structure 4:339-50.

62. Bommer M, Kunze C, Fesseler J, Schubert T, Diekert G, Dobbek H. 2014. Structural basis for organohalide respiration. Science 346:455-8.

63. Drennan CL, Huang S, Drummond JT, Matthews RG, Ludwig ML. 1994. How a protein binds $B_{12}$ : $A$ 3.0 A X-ray structure of $B_{12}$-binding domains of methionine synthase. Science 266:1669-74.

64. Gruber K, Reitzer R, Kratky C. 2001. Radical shuttling in a protein: ribose pseudorotation controls alkyl-radical transfer in the coenzyme $B_{12}$ dependent enzyme glutamate mutase. Angew Chem Int Ed Engl 40:3377-3380.

65. Berkovitch F, Behshad E, Tang KH, Enns EA, Frey PA, Drennan CL. 2004. A locking mechanism preventing radical damage in the absence of substrate, as revealed by the x-ray structure of lysine 5,6-aminomutase. Proc Natl Acad Sci U S A 101:15870-5.

66. Wolthers KR, Levy C, Scrutton NS, Leys D. 2010. Large-scale domain dynamics and adenosylcobalamin reorientation orchestrate radical catalysis in ornithine 4,5aminomutase. J Biol Chem 285:13942-50.

67. Jost M, Cracan V, Hubbard PA, Banerjee R, Drennan CL. 2015. Visualization of a radical $B_{12}$ enzyme with its G-protein chaperone. Proc Natl Acad Sci U S A 112:2419-24.

68. Payne KA, Fisher K, Sjuts H, Dunstan MS, Bellina B, Johannissen L, Barran P, Hay S, Rigby SE, Leys D. 2015. Epoxyqueuosine reductase structure suggests a mechanism for cobalamin-dependent tRNA modification. J Biol Chem 290:27572-81. 
69. Payne KA, Quezada CP, Fisher K, Dunstan MS, Collins FA, Sjuts H, Levy C, Hay S, Rigby SE, Leys D. 2015. Reductive dehalogenase structure suggests a mechanism for $\mathrm{B}_{12}$-dependent dehalogenation. Nature 517:513-516.

70. Kurteva-Yaneva N, Zahn M, Weichler MT, Starke R, Harms H, Muller RH, Strater N, Rohwerder T. 2015. Structural basis of the stereospecificity of bacterial $\mathrm{B}_{12}$-dependent 2hydroxyisobutyryl-CoA mutase. J Biol Chem 290:9727-37.

71. Tanioka Y, Miyamoto E, Yabuta Y, Ohnishi K, Fujita T, Yamaji R, Misono H, Shigeoka S, Nakano Y, Inui H, Watanabe F. 2010. Methyladeninylcobamide functions as the cofactor of methionine synthase in a cyanobacterium, Spirulina platensis NIES-39. FEBS Lett 584:3223-6.

72. Lengyel P, Mazumder R, Ochoa S. 1960. Mammalian methylmalonyl isomerase and vitamin $B_{12}$ coenzymes. Proceedings of the National Academy of Sciences of the United States of America 46:1312-1318.

73. Barker HA, Smyth RD, Weissbach H, Toohey JI, Ladd JN, Volcani BE. 1960. Isolation and properties of crystalline cobamide coenzymes containing benzimidazole or 5,6dimethylbenzimidazole. J Biol Chem 235:480-8.

74. Dowling DP, Croft AK, Drennan CL. 2012. Radical use of Rossmann and TIM barrel architectures for controlling coenzyme $B_{12}$ chemistry. Annu Rev Biophys 41:403-27.

75. Hosotani K, Yokota A, Nakano Y, Kitaoka S. 1980. The metabolic pathway of propionate in Euglena gracilis z grown under illumination. Agricultural and Biological Chemistry 44:1097-1103.

76. Savvi S, Warner DF, Kana BD, McKinney JD, Mizrahi V, Dawes SS. 2008. Functional characterization of a vitamin $\mathrm{B}_{12}$-dependent methylmalonyl pathway in Mycobacterium tuberculosis: Implications for propionate metabolism during growth on fatty acids. Journal of Bacteriology 190:3886.

77. Fenton WA, Gravel RA, Rosenblatt DS. 2014. Disorders of propionate and methylmalonate metabolism. In Beaudet AL, Vogelstein B, Kinzler KW, Antonarakis SE, Ballabio A, Gibson KM, Mitchell G (ed), The Online Metabolic and Molecular Bases of Inherited Disease. The McGraw-Hill Companies, Inc., New York, NY.

78. Charles TC, Cai GQ, Aneja P. 1997. Megaplasmid and chromosomal loci for the PHB degradation pathway in Rhizobium (Sinorhizobium) meliloti. Genetics 146:1211-20.

79. Hunaiti AA, Kolattukudy PE. 1984. Source of methylmalonyl-coenzyme A for erythromycin synthesis: Methylmalonyl-coenzyme A mutase from Streptomyces erythreus. Antimicrob Agents Chemother 25:173-8.

80. Han Y, Hawkins AS, Adams MW, Kelly RM. 2012. Epimerase (Msed_0639) and mutase (Msed_0638 and Msed_2055) convert (S)-methylmalonyl-coenzyme A (CoA) to succinylCoA in the Metallosphaera sedula 3-hydroxypropionate/4-hydroxybutyrate cycle. Appl Environ Microbiol 78:6194-202.

81. Aldor IS, Kim SW, Prather KL, Keasling JD. 2002. Metabolic engineering of a novel propionate-independent pathway for the production of poly(3-hydroxybutyrate-co-3- 
hydroxyvalerate) in recombinant Salmonella enterica serovar Typhimurium. Appl Environ Microbiol 68:3848-54.

82. Gonzalez-Garcia RA, McCubbin T, Wille A, Plan M, Nielsen LK, Marcellin E. 2017. Awakening sleeping beauty: production of propionic acid in Escherichia coli through the sbm operon requires the activity of a methylmalonyl-CoA epimerase. Microb Cell Fact 16:121.

83. Gross F, Ring MW, Perlova O, Fu J, Schneider S, Gerth K, Kuhlmann S, Stewart AF, Zhang Y, Muller R. 2006. Metabolic engineering of Pseudomonas putida for methylmalonyl-CoA biosynthesis to enable complex heterologous secondary metabolite formation. Chem Biol 13:1253-64.

84. Reeves AR, Brikun IA, Cernota WH, Leach BI, Gonzalez MC, Weber JM. 2007. Engineering of the methylmalonyl-CoA metabolite node of Saccharopolyspora erythraea for increased erythromycin production. Metab Eng 9:293-303.

85. Dayem LC, Carney JR, Santi DV, Pfeifer BA, Khosla C, Kealey JT. 2002. Metabolic engineering of a methylmalonyl-CoA mutase-epimerase pathway for complex polyketide biosynthesis in Escherichia coli. Biochemistry 41:5193-201.

86. Li J, Zhu X, Chen J, Zhao D, Zhang X, Bi C. 2017. Construction of a novel anaerobic pathway in Escherichia coli for propionate production. BMC biotechnology 17:38-38.

87. Srirangan K, Akawi L, Liu X, Westbrook A, Blondeel EJ, Aucoin MG, Moo-Young M, Chou CP. 2013. Manipulating the sleeping beauty mutase operon for the production of 1-propanol in engineered Escherichia coli. Biotechnol Biofuels 6:139.

88. Akawi L, Srirangan K, Liu X, Moo-Young M, Perry Chou C. 2015. Engineering Escherichia coli for high-level production of propionate. J Ind Microbiol Biotechnol 42:1057-1072.

89. Chowdhury S, Banerjee R. 1999. Role of the dimethylbenzimidazole tail in the reaction catalyzed by coenzyme $\mathrm{B}_{12}$-dependent methylmalonyl-CoA mutase. Biochemistry 38:15287-94.

90. De Hertogh AA, Mayeux PA, Evans HJ. 1964. The relationship of cobalt requirement to propionate metabolism in Rhizobium. Journal of Biological Chemistry 239:2446-2453.

91. Charles TC, Aneja P. 1999. Methylmalonyl-CoA mutase encoding gene of Sinorhizobium meliloti. Gene 226:121-7.

92. Miyamoto E, Watanabe F, Charles TC, Yamaji R, Inui H, Nakano Y. 2003. Purification and characterization of homodimeric methylmalonyl-CoA mutase from Sinorhizobium meliloti. Arch Microbiol 180:151-4.

93. Haller T, Buckel T, Rétey J, Gerlt JA. 2000. Discovering new enzymes and metabolic pathways: Conversion of succinate to propionate by Escherichia coli. Biochemistry 39:4622-4629.

94. Kliewer M, Evans HJ. 1963. Identification of cobamide coenzyme in nodules of symbionts \& isolation of the $\mathrm{B}_{12}$ coenzyme from Rhizobium meliloti. Plant Physiol 38:559. 
95. Hazra AB, Han AW, Mehta AP, Mok KC, Osadchiy V, Begley TP, Taga ME. 2015. Anaerobic biosynthesis of the lower ligand of vitamin $B_{12}$. Proc Natl Acad Sci U S A 112:10792-7.

96. Crofts TS, Seth EC, Hazra AB, Taga ME. 2013. Cobamide structure depends on both lower ligand availability and CobT substrate specificity. Chem Biol 20:1265-74.

97. Froese DS, Kochan G, Muniz JR, Wu X, Gileadi C, Ugochukwu E, Krysztofinska E, Gravel RA, Oppermann U, Yue WW. 2010. Structures of the human GTPase MMAA and vitamin $\mathrm{B}_{12}$-dependent methylmalonyl-CoA mutase and insight into their complex formation. J Biol Chem 285:38204-13.

98. Miyamoto E, Tanioka Y, Nishizawa-Yokoi A, Yabuta Y, Ohnishi K, Misono H, Shigeoka S, Nakano Y, Watanabe F. 2010. Characterization of methylmalonyl-CoA mutase involved in the propionate photoassimilation of Euglena gracilis Z. Arch Microbiol 192:437-46.

99. Miyamoto E, Watanabe F, Yamaji R, Inui H, Sato K, Nakano Y. 2002. Purification and characterization of methylmalonyl-CoA mutase from a methanol-utilizing bacterium, Methylobacterium extorquens NR-1. J Nutr Sci Vitaminol (Tokyo) 48:242-6.

100. Birch A, Leiser A, Robinson JA. 1993. Cloning, sequencing, and expression of the gene encoding methylmalonyl-coenzyme A mutase from Streptomyces cinnamonensis. J Bacteriol 175:3511-9.

101. Zhang W, Yang L, Jiang W, Zhao G, Yang Y, Chiao J. 1999. Molecular analysis and heterologous expression of the gene encoding methylmalonyl-coenzyme A mutase from rifamycin SV-producing strain Amycolatopsis mediterranei U32. Applied Biochemistry and Biotechnology 82:209-225.

102. Yabuta Y, Kamei Y, Bito T, Arima J, Yoneda K, Sakuraba H, Ohshima T, Nakano Y, Watanabe F. 2015. Functional and structural characteristics of methylmalonyl-CoA mutase from Pyrococcus horikoshii. Biosci Biotechnol Biochem 79:710-7.

103. Chowdhury S, Thomas MG, Escalante-Semerena JC, Banerjee R. 2001. The coenzyme $\mathrm{B}_{12}$ analog 5'-deoxyadenosylcobinamide-GDP supports catalysis by methylmalonyl-CoA mutase in the absence of trans-ligand coordination. J Biol Chem 276:1015-9.

104. Kanehisa M, Goto S. 2000. KEGG: kyoto encyclopedia of genes and genomes. Nucleic Acids Res 28:27-30.

105. Borths EL, Poolman B, Hvorup RN, Locher KP, Rees DC. 2005. In vitro functional characterization of BtuCD-F, the Escherichia coli $\mathrm{ABC}$ transporter for vitamin $\mathrm{B}_{12}$ uptake. Biochemistry 44:16301-9.

106. Heller K, Mann BJ, Kadner RJ. 1985. Cloning and expression of the gene for the vitamin $\mathrm{B}_{12}$ receptor protein in the outer membrane of Escherichia coli. J Bacteriol 161:896-903.

107. Hazra Amrita B, Tran Jennifer LA, Crofts Terence S, Taga Michiko E. 2013. Analysis of substrate specificity in CobT homologs reveals widespread preference for DMB, the lower axial ligand of vitamin $B_{12}$. Chemistry \& Biology 20:1275-1285. 
108. Mukherjee S, Das Mandal S, Gupta N, Drory-Retwitzer M, Barash D, Sengupta S. 2019. RiboD: A comprehensive database for prokaryotic riboswitches. Bioinformatics doi:10.1093/bioinformatics/btz093.

109. Nahvi A, Barrick JE, Breaker RR. 2004. Coenzyme $B_{12}$ riboswitches are widespread genetic control elements in prokaryotes. Nucleic Acids Res 32:143-50.

110. Widner FJ, Lawrence AD, Deery E, Heldt D, Frank S, Gruber K, Wurst K, Warren MJ, Krautler B. 2016. Total synthesis, structure, and biological activity of adenosylrhodibalamin, the non-natural rhodium homologue of coenzyme $B_{12}$. Angew Chem Int Ed Engl 55:11281-6.

111. Calafat AM, Taoka S, Puckett JM, Semerad C, Yan H, Luo L, Chen H, Banerjee R, Marzilli LG. 1995. Structural and electronic similarity but functional difference in methylmalonyl-CoA mutase between coenzyme $B_{12}$ and the analog 2',5'dideoxyadenosylcobalamin. Biochemistry 34:14125-14130.

112. Fukuoka M, Nakanishi Y, Hannak RB, Krautler B, Toraya T. 2005. Homoadenosylcobalamins as probes for exploring the active sites of coenzyme $\mathrm{B}_{12}$ dependent diol dehydratase and ethanolamine ammonia-lyase. Febs j 272:4787-96.

113. Santos JA, Rempel S, Mous STM, Pereira CT, ter Beek J, de Gier J-W, Guskov A, Slotboom DJ. 2018. Functional and structural characterization of an ECF-type ABC transporter for vitamin $\mathrm{B}_{12}$. eLife 7:e35828.

114. Rodionov DA, Hebbeln P, Eudes A, ter Beek J, Rodionova IA, Erkens GB, Slotboom DJ, Gelfand MS, Osterman AL, Hanson AD, Eitinger T. 2009. A novel class of modular transporters for vitamins in prokaryotes. Journal of Bacteriology 191:42.

115. Johnson CL, Pechonick E, Park SD, Havemann GD, Leal NA, Bobik TA. 2001. Functional genomic, biochemical, and genetic characterization of the Salmonella pduO gene, an ATP:cob(I)alamin adenosyltransferase gene. J Bacteriol 183:1577-84.

116. Escalante-Semerena JC, Suh SJ, Roth JR. 1990. cobA function is required for both de novo cobalamin biosynthesis and assimilation of exogenous corrinoids in Salmonella typhimurium. Journal of Bacteriology 172:273-280.

117. Abreu NA, Taga ME. 2016. Decoding molecular interactions in microbial communities. FEMS Microbiol Rev 40:648-63.

118. Degnan PH, Taga ME, Goodman AL. 2014. Vitamin $B_{12}$ as a modulator of gut microbial ecology. Cell Metab 20:769-778.

119. Seth EC, Taga ME. 2014. Nutrient cross-feeding in the microbial world. Front Microbiol 5:350.

120. Yan J, Ritalahti KM, Wagner DD, Loffler FE. 2012. Unexpected specificity of interspecies cobamide transfer from Geobacter spp. to organohalide-respiring Dehalococcoides mccartyi strains. Appl Environ Microbiol 78:6630-6.

121. Ma AT, Beld J, Brahamsha B. 2017. An amoebal grazer of cyanobacteria requires cobalamin produced by heterotrophic bacteria. Applied and Environmental Microbiology 83:e00035-17. 
122. Belzer C, Chia LW, Aalvink S, Chamlagain B, Piironen V, Knol J, de Vos WM. 2017. Microbial metabolic networks at the mucus layer lead to diet-independent butyrate and vitamin $\mathrm{B}_{12}$ production by intestinal symbionts. mBio 8:e00770-17.

123. Heal KR, Qin W, Ribalet F, Bertagnolli AD, Coyote-Maestas W, Hmelo LR, Moffett JW, Devol AH, Armbrust EV, Stahl DA, Ingalls AE. 2017. Two distinct pools of $B_{12}$ analogs reveal community interdependencies in the ocean. Proc Natl Acad Sci U S A 114:364369.

124. Fortier A, Grosdidier A, Hernandez C, Baratin D, Kuznetsov D, de Castro E, Gasteiger E, Csardi G, Rossier G, Stockinger H, Xenarios I, Mostaguir K, Arnold K, Jonnalagedda M, Redaschi N, Artimo P, Liechti R, Moretti S, Duvaud S, loannidis V, Flegel V. 2012. ExPASy: SIB bioinformatics resource portal. Nucleic Acids Research 40:W597-W603.

125. Crosby HA, Rank KC, Rayment I, Escalante-Semerena JC. 2012. Structure-guided expansion of the substrate range of methylmalonyl coenzyme A synthetase (MatB) of Rhodopseudomonas palustris. Appl Environ Microbiol 78:6619-29.

126. Hoffmann B, Oberhuber M, Stupperich E, Bothe H, Buckel W, Konrat R, Kräutler B. 2000. Native corrinoids from Clostridium cochlearium are adeninylcobamides: Spectroscopic analysis and identification of pseudovitamin $\mathrm{B}_{12}$ and factor A. J Bacteriol 182:4773-82.

127. Crofts TS, Hazra AB, Tran JL, Sokolovskaya OM, Osadchiy V, Ad O, Pelton J, Bauer S, Taga ME. 2014. Regiospecific formation of cobamide isomers is directed by CobT. Biochemistry 53:7805-15.

128. Brown KL, Zou X. 1999. Thermolysis of coenzymes $B_{12}$ at physiological temperatures: activation parameters for cobalt-carbon bond homolysis and a quantitative analysis of the perturbation of the homolysis equilibrium by the ribonucleoside triphosphate reductase from Lactobacillus leichmannii. J Inorg Biochem 77:185-95.

129. Hogenkamp HPC. 1975. The chemistry of cobalamins and related compounds, p 55. In Babior BM (ed), Cobalamin Biochemistry and Pathophysiology. John Wiley and Sons, Inc.

130. Schneider Z, Stroinski A. 1987. Comprehensive $B_{12}$ : Chemistry, Biochemistry, Nutrition, Ecology, Medicine. In Gruyter Wd (ed).

131. Stupperich E, Eisinger HJ, Krautler B. 1988. Diversity of corrinoids in acetogenic bacteria. p-cresolylcobamide from Sporomusa ovata, 5-methoxy-6-

methylbenzimidazolylcobamide from Clostridium formicoaceticum and vitamin $\mathrm{B}_{12}$ from Acetobacterium woodii. Eur J Biochem 172:459-64.

132. Warner JR, Copley SD. 2007. Pre-steady-state kinetic studies of the reductive dehalogenation catalyzed by tetrachlorohydroquinone dehalogenase. Biochemistry 46:13211-22.

133. Guex N, Peitsch MC, Schwede T. 2009. Automated comparative protein structure modeling with SWISS-MODEL and Swiss-PdbViewer: a historical perspective. Electrophoresis 30 Suppl 1:S162-73. 
134. Schrödinger Release 2015: Maestro S, LLC, New York, NY, 2015.

135. Schrödinger Release 2015: MacroModel S, LLC, New York, NY, 2015.

136. Taoka S, Padmakumar R, Lai MT, Liu HW, Banerjee R. 1994. Inhibition of the human methylmalonyl-CoA mutase by various CoA-esters. J Biol Chem 269:31630-4.

137. Campbell GR, Taga ME, Mistry K, Lloret J, Anderson PJ, Roth JR, Walker GC. 2006. Sinorhizobium meliloti bluB is necessary for production of 5,6-dimethylbenzimidazole, the lower ligand of $B_{12}$. Proc Natl Acad Sci U S A 103:4634-9.

138. Banerjee RV, Johnston NL, Sobeski JK, Datta P, Matthews RG. 1989. Cloning and sequence analysis of the Escherichia coli metH gene encoding cobalamin-dependent methionine synthase and isolation of a tryptic fragment containing the cobalamin-binding domain. J Biol Chem 264:13888-95.

139. Sato K, Inukai S, Shimizu S. 1974. Vitamin $B_{12}$-dependent methionine synthesis in Rhizobium meliloti. Biochem Biophys Res Commun 60:723-8.

140. Cowles JR, Evans HJ, Russell SA. 1969. B 12 coenzyme-dependent ribonucleotide reductase in Rhizobium species and the effects of cobalt deficiency on the activity of the enzyme. J Bacteriol 97:1460-5.

141. Taga ME, Walker GC. 2010. Sinorhizobium meliloti requires a cobalamin-dependent ribonucleotide reductase for symbiosis with its plant host. Mol Plant Microbe Interact 23:1643-54.

142. Maniatis T, Fritsch EF, Sambrook J. 1989. Molecular cloning: a laboratory manual. Cold spring harbor laboratory press. 